\title{
Simplifying the Performance Analysis of the SPRT for GPS Acquisition
}

\author{
Niamh O'Mahony, ${ }^{1}$ Gérard Lachapelle, ${ }^{2}$ and Colin C. Murphy ${ }^{1}$ \\ ${ }^{1}$ Department of Electrical and Electronic Engineering, University College Cork, College Rd., Cork, Ireland \\ ${ }^{2}$ PLAN Group, Departement of Geomatics Engineering, University of Calgary, 2500 University Drive NW, Calgary, \\ $A B$, Canada T2N $1 N 4$
}

Correspondence should be addressed to Niamh O’Mahony, niamhom@rennes.ucc.ie

Received 18 August 2010; Revised 7 December 2010; Accepted 13 February 2011

Academic Editor: Abbas Mohammed

Copyright ( 2011 Niamh O’Mahony et al. This is an open access article distributed under the Creative Commons Attribution License, which permits unrestricted use, distribution, and reproduction in any medium, provided the original work is properly cited.

A new approximation for the distribution of the probability ratio in a sequential probability ratio test (SPRT) using noncoherent integration across a full code period is presented. The new approximation is valid for the carrier-to-noise power ratios $\left(C / N_{0}\right)$ typically encountered in GPS acquisition $\left(20 \mathrm{~dB}-\mathrm{Hz} \leq \mathrm{C} / \mathrm{N}_{0} \leq 50 \mathrm{~dB}-\mathrm{Hz}\right)$, and it allows accurate theoretical performance analysis of the SPRT to be carried out for signals in this $C / N_{0}$ range, eliminating the need for lengthy simulations for each scenario under investigation. Thus, the SPRT performance can be readily compared to that of other acquisition strategies for receiver design. Previous approximations in the literature are not valid in the range $20 \mathrm{~dB}-\mathrm{Hz} \leq C / N_{0} \leq 50 \mathrm{~dB}-\mathrm{Hz}$.

\section{Introduction}

Acquisition of direct-sequence spread spectrum (DS/SS) code division multiple access (CDMA) signals, such as the Global Positioning System (GPS) L1 C/A signals [1], involves synchronising the received signal with a locally generated replica signal. Synchronisation is carried out in two dimensions, namely, code phase, $\zeta$, and Doppler uncertainty frequency, $\omega_{D}$. The received signal is correlated with the local replica signal at each code phase and Doppler estimate, and the outputs of the correlator are combined to make a decision that the signal is present and correctly synchronised (an $H_{1}$ decision) or that the signal is not present or not synchronised (an $H_{0}$ decision). Each estimate, $\hat{\theta}=\left\{\hat{\zeta}, \widehat{\omega}_{D}\right\}$, is referred to as a "cell" in the search space. The decision making procedure or "search-control strategy" can be based on a fixed or variable number of correlator outputs, and its goal is to make a correct decision in the minimum time. Thus, the main performance metrics are the time required for a decision to be made (the dwell time) in an $H_{1}$ or an $H_{0}$ cell, $\tau_{1}$ or $\tau_{0}$, respectively, and the probabilities of correct detection, $P_{d c}$, and false alarm, $P_{f a c}$ (the subscript " $c$ " denotes the "cell level" probabilities).
Wald's sequential probability ratio test (SPRT) [2] is known to be the optimal strategy in terms of simultaneously minimising the mean dwell time in both $H_{1}$ and $H_{0}$ cells, $\bar{\tau}_{1}$ and $\bar{\tau}_{0}$, respectively, for a particular performance pair, $\left\{P_{d c}, P_{f a c}\right\}$. The SPRT is widely used throughout the field of communications, with recent applications in many areas, for example, radar detection [3], spectrum sensing for cognitive radio [4], and CDMA acquisition [5-7]. The SPRT is also commonly used as a benchmark for comparing the acquisition performance of CDMA systems, for example, comparing the performances of different code families [8] or the performance of a system under varying fading conditions [9].

Despite its optimality condition and widespread use across the communications field, the SPRT is not widely reported for the acquisition of GPS signals. This might be due, in part, to its computational complexity (which shall be discussed later) or to the fact that its optimality in a $H_{1}$ cell is limited to the case when the received carrier-to-noise power ratio, $C / N_{0}^{T}$, equals that for which the receiver was designed, $C / N_{0}^{D}$, with a large increase in $\bar{\tau}_{1}$ and a decrease in $P_{d c}$ occurring for $C / N_{0}^{T}<C / N_{0}^{D}$. However, a recent study by the authors [10] has shown promising results regarding the SPRT 
performance for GPS L1 C/A signal acquisition, compared to that of a commonly used single dwell detector (SDD). These results are applicable in particular to the case when the SPRT is used in the verification stage of acquisition, when a quick initial search throughout the search space has selected a small number of potential candidate cells. We have shown in [10], through simulation, that, for certain target performances, the worst case dwell time for the SPRT becomes as good as that of the SDD, when the $C / N_{0}$ becomes weak.

The motivation of this work is to theoretically verify the accuracy of the simulated results, presented in [10], and to extend our previous study by analysing the SPRT performance across a wide range of $C / N_{0}$ values and target probabilities of detection and false alarm to investigate the range of conditions under which the SPRT is, indeed, a desirable choice of search-control strategy. The results of this study allow the SPRT performance to be compared with that of other suboptimal strategies, for example, the coincidence detector [11] or up-down counter [12, 13], so that important receiver design decisions regarding the trade-off between performance and computational complexity can be made. An example of such a comparison can be found in [13]. For such a study, simulation alone is not practical, especially for weak signals, which require long dwell times and, hence, long simulation times. Thus, tractable theoretical expressions for the dwell time and the probabilities of detection and false alarm are required. Typically, for very weak signals, approximations due to Wald [2] are used for the average number of samples required to make a decision (ASN) (which is directly proportional to the dwell time) and for the probabilities of detection and false alarm [7, 14, 15]. However, the carrier-to-noise ratios typically encountered in GPS acquisition, ranging from "harsh environments" to "open-sky" conditions, $20 \mathrm{~dB}-\mathrm{Hz} \leq C / N_{0} \leq 50 \mathrm{~dB}-\mathrm{Hz}$, respectively, are larger than the range of validity of Wald's approximations and, so, the approximations result in highly inaccurate performance predictions.

This paper presents a new, simplified approximation for the distribution of the probability ratio to address the need for an accurate and tractable analysis for the SPRT performance in GPS L1 C/A acquisition, in order that comparisons with other strategies can readily be made. This eliminates the need for lengthy simulations, allowing a wide range of scenarios, in terms of varying $C / N_{0}$ values, attenuation effects, and target probabilities of detection and false alarm, to be evaluated efficiently. The operation of the SPRT is briefly described in Section 2. In Section 3, we outline the proposed approximation and provide expressions for its parameters, as functions of the signal parameters. Validation of the approximation is illustrated in Section 4, highlighting the improved accuracy of the new approximation relative to previous approximations. It is also shown that evaluation of performance metrics using the new approximation requires only $1 \%$ of the computation time for existing numerical methods using the same computer. Data collected from live GPS satellites is presented in Section 5, illustrating the accuracy of the theoretical results. The performance of the SPRT is theoretically investigated in Section 6 and compared with that of common suboptimal strategies. The impact on performance of unknown power levels is also addressed. Finally, some concluding remarks are presented in Section 7.

\section{SPRT Operation and Performance}

Operation of the SPRT is based on comparison with upper and lower thresholds, $A$ and $B$, respectively, of the cumulative ratio, $Z_{n}$, of the probabilities of obtaining the given set of samples, $\mathbf{x}_{n}=\left\{x_{1}, \ldots, x_{n}\right\}$, under the $H_{1}$ hypothesis and the $H_{0}$ hypothesis at the $n$th trial, $n=1,2, \ldots$ [2]. The cumulative probability ratio at the $n$th trial is given by

$$
Z_{n}=\sum_{k=1}^{n} z_{k}
$$

where $z_{k}$ is the single trial probability ratio at the $k$ th trial, given by

$$
z_{k}=\ln \left(\frac{f_{H_{1}}\left(x_{k}, \lambda\right)}{f_{H_{0}}\left(x_{k}, \lambda\right)}\right),
$$

where $f_{H_{i}}\left(x_{k}, \lambda\right)$ is the probability distribution function (pdf) of the correlator output under the $H_{i}$ hypothesis, $i=0,1$, and $\lambda$ is a parameter representing the $C / N_{0}$; the natural logarithm is taken for convenience in the manner of [2]. For the well-known noncoherent combining detector (NCCD), which is commonly used for the acquisition of CDMA signals in the presence of unknown Doppler frequency and data modulation (e.g., GPS signals) [15], with noncoherent integration over $K=1$ code periods, the probability ratio is given by

$$
z_{k}=-\lambda+\ln \left(I_{0}\left(2 \sqrt{\lambda x_{k}}\right)\right),
$$

where $I_{\nu}(x)$ is the $v$ th order modified Bessel function of the 1st kind [16, Equation 9.6.10], and it should be noted that the raw correlator output has been appropriately normalised to yield unit variance in $x_{k}$. During each trial, $Z_{n}$ is compared to the thresholds, $A$ and $B$, and a decision made as follows:

$$
\begin{aligned}
& B<Z_{n}<A \Longrightarrow \text { Take another sample, } \\
& Z_{n} \leq B \Longrightarrow \text { Terminate the test, accepting } H_{0}, \\
& Z_{n} \geq A \Longrightarrow \text { Terminate the test, accepting } H_{1} .
\end{aligned}
$$

The choice $A=\ln \left(P_{d c} / P_{f a c}\right)$ and $B=\ln \left(\left(1-P_{d c}\right) /(1-\right.$ $\left.P_{f a c}\right)$ ), suggested by Wald [2], is typical in the literature, even though this usually results in a larger probability of correct detection and smaller probability of false alarm than the target probabilities for which the test was designed, with a slight increase in mean dwell time over that required for the exact target performance. This is particularly true for strong signals.

When both the mean and variance of $z$ converge to zero, approximations for the operating characteristic function (OCF) (the probability of terminating the test with an $H_{0}$ decision) and average sample number (ASN), due to Wald [2, Equations 3:43 and 3:57], respectively, can be used to 
determine the SPRT performance. However, as illustrated in Figures 1 (a) and $1(\mathrm{~b})$, for $\mathrm{C} / \mathrm{N}_{0}>25 \mathrm{~dB}-\mathrm{Hz}$ (approximately), these convergence conditions are not satisfied and, so, Wald's approximations are not valid for GPS acquisition performance.

Methods of evaluating the exact ASN and OCF have long existed, for example, Wald and Girshick's method based on characteristic functions [2, Appendix A.4] and Proakis' Markov chain method [17], either of which can be employed for the GPS case. Both of these methods require the calculation of the probability, $h_{i}$, that $z$ equals an integer, $i$, times a positive constant, $d$, (i.e., $h_{i}=\operatorname{Pr}(z=i d)$ ), for $i=\left\lfloor z_{\min } / d\right\rfloor, \ldots,\left\lceil z_{\max } / d\right\rceil$, where $z_{\min }$ and $z_{\max }$ are the minimum and maximum values of $z$, respectively, the $\lfloor\cdot\rfloor$ function returns the largest integer which is less than or equal to its argument, and the $\lceil\cdot\rceil$ function returns the smallest integer which is greater than or equal to its argument. For continuously distributed $z$, as is the case here, the distribution can be approximated by a discrete one by choosing a suitably small value of $d$, and $h_{i}$ is given by

$$
h_{i}=\int_{(i-0.5) d}^{(i+0.5) d} f_{z}\left(z, \lambda_{d}, \lambda_{t}\right) d z
$$

where $f_{z}\left(z, \lambda_{d}, \lambda_{t}\right)$ is the pdf of $z$ conditioned on the design point $C / N_{0}$ parameter, $\lambda_{d}$, and the true $C / N_{0}$ parameter, $\lambda_{t}$. Typical $C / N_{0}$ estimators require synchronisation to have been achieved in Doppler and code phase domains, for example, $[18,19]$, and so are not feasible before acquisition. Despite this fact, the SPRT implementation requires an estimate of $C / N_{0}^{T}$ to be determined-this is the design point, $C / N_{0}^{D}$, which will be seen to affect the performance of the detector, in terms of detection probability and mean dwell time. In practice, if a signal is not acquired at a particular value of $C / N_{0}^{D}$, a subsequent test with a lower design point can be carried out, and this process iterated until a suitable estimate of $C / N_{0}^{D}$ is found to yield an acquisition decision. Of course, constant false alarm rate is often chosen as the design criterion, for example, [20], in which case the $C / N_{0}^{D}$ is not the important design criterion for any strategy. However, if a particular cell level detection probability is desired, then considering the expected $C / N_{0}$ will improve the detection probability and, hence, the overall performance. This effect will be illustrated later in the work by means of results describing the detection performance in the presence of attenuation between $C / N_{0}^{D}$ and $C / N_{0}^{T}$.

Albert's integral equations [21] can also be used to analyse the performance of a general class of sequential tests, including the SPRT [22]. For example, the probability, $P_{H_{1}}\left(z_{0}\right)$, of terminating the test in an $H_{1}$ cell, given an initial probability ratio value of $z_{0}$, is defined by the following Fredholm integral equation of the second kind [21]:

$$
\begin{aligned}
P_{H_{1}}\left(z_{0}\right)= & \int_{A}^{\infty} f_{z}\left(z-z_{0}, \lambda_{d}, \lambda_{t}\right) d z \\
& +\int_{B}^{A} P_{H_{1}}\left(z_{1}\right) f_{z}\left(z_{1}-z_{0}, \lambda_{d}, \lambda_{t}\right) d z_{1},
\end{aligned}
$$

with similar expressions available for the moments of the ASN. In the absence of analytical forms for the integrals (which are usually not available [22]), various numerical methods exist for the solution of the above equations, including the polynomial interpolation method of [22]. Other sequential tests, such as the biased sequential squarelaw detector, which approximates the SPRT under very weak signal conditions, can be used to simplify the analysis, but the performance of such tests is suboptimal $[23,24]$. The focus of this work is on the optimality of the SPRT performance for GPS signals, so approximate tests are not considered.

Currently, an expression for the pdf of $z$, in terms of $z$ itself, is not available, to the best of the authors' knowledge, so (5), (6), and similar ones cannot be solved directly. However, expressions for the pdf of the correlator output, $x$, in $H_{1}$ and $H_{0}$ cells, and an expression for the probability ratio, as a function of $x,(3)$, are known. Much effort has been applied to manipulating these known expressions to find an expression for $f_{z}\left(z, \lambda_{d}, \lambda_{t}\right)$ in an integrable form, with a power series expansion yielding the most promising results. This work is outlined in Appendix A. Unfortunately, for the $C / N_{0}$ range of interest for GPS, the resulting power series is not convergent and, so, it is not practical for GPS acquisition analysis. Hence, a new approximation for $f_{z}\left(z, \lambda_{d}, \lambda_{t}\right)$ is sought.

\section{Approximation for the Distribution of the Probability Ratio}

In the absence of accurate analytical expressions for the performance metrics, a practical approach is to turn to alternative methods, such as numerical methods and model fitting. By examining the shape of the distribution of $z$ for varying $\lambda_{d}$ and $\lambda_{t}$, it has been observed that $f_{z}\left(z, \lambda_{d}, \lambda_{t}\right)$ can be very closely approximated by a truncated normal distribution, with one-sided lower truncation point, $t_{l}=-\lambda_{d}$, whose pdf is given by

$$
f_{\mathrm{TN}}\left(z, \mu, \sigma, t_{l}\right)=\frac{\phi((z-\mu) / \sigma)}{1-\Phi\left(\left(t_{l}-\mu\right) / \sigma\right)}
$$

where $\phi((z-\mu) / \sigma)$ and $\Phi((z-\mu) / \sigma)$ are, respectively, the probability distribution function and cumulative distribution function of the normal distribution with mean, $\mu$, and standard deviation, $\sigma$. The closeness of the fit is illustrated in Figures 2 and 3, which show Monte Carlo simulation outputs and truncated normal distributions for suitably estimated values of $\mu$ and $\sigma$. A number of cases are illustrated in the figures to show the effect of varying the design point, $C / N_{0}^{D}$, and the mismatch between the true carrier-to-noise ratio, $C / N_{0}^{T}$, and $C / N_{0}^{D}, a=C / N_{0}^{T}-C / N_{0}^{D}=10 \log _{10}\left(\lambda_{t} / \lambda_{d}\right)$. The Monte Carlo simulations consisted of generating $10^{5}$ sample probability ratio values per data point (i.e., per value of $C / N_{0}^{D}$ and $a$ ), where the probability ratios were generated from simulated GPS data, including data modulation, code phase and Doppler frequency components, and randomly generated noise for each sample yielding the appropriate value of $C / N_{0}^{T}$. A histogram for each set of $10^{5}$ samples was constructed and converted to a probability density 


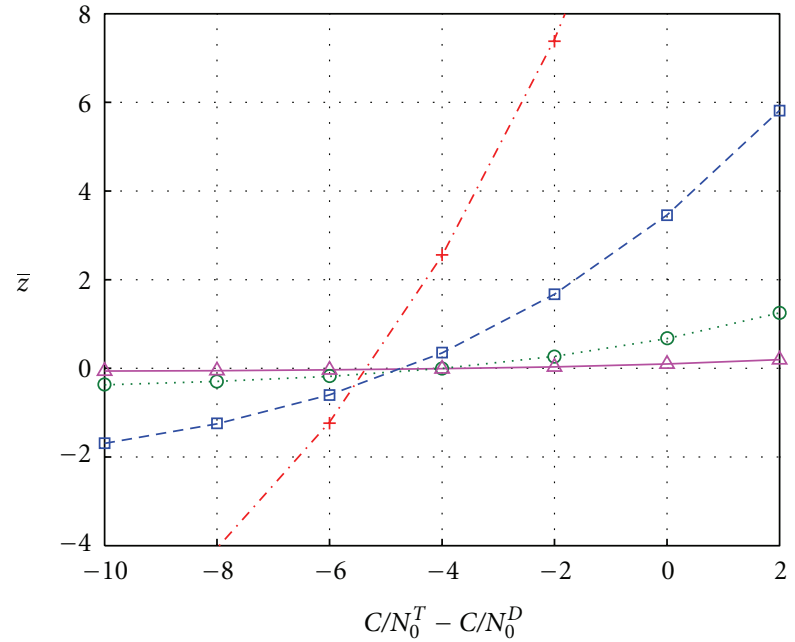

$\begin{array}{ll}- & 27 \mathrm{~dB}-\mathrm{Hz} \\ \ldots . & 32 \mathrm{~dB}-\mathrm{Hz} \\ --- & 37 \mathrm{~dB}-\mathrm{Hz} \\ -.- & 42 \mathrm{~dB}-\mathrm{Hz}\end{array}$

(a) Mean

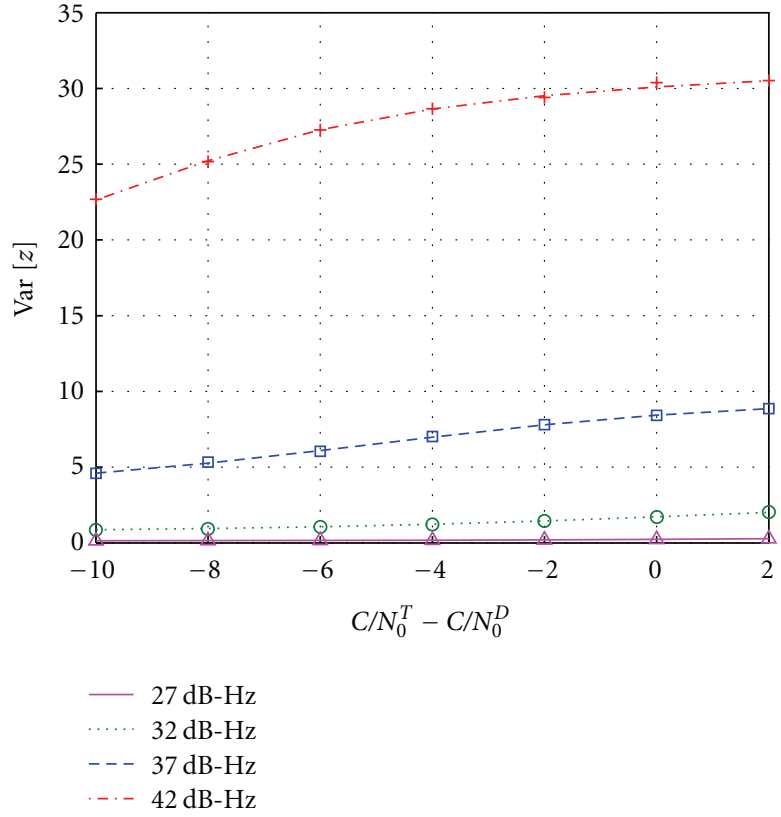

(b) Variance

FIGURE 1: Mean (a) and variance (b) of single trial probability ratio versus attenuation: theoretical (lines) and simulated (symbols); $C / N_{0}^{D}(\mathrm{~dB}-\mathrm{Hz})=42(+), 37(\square), 32(\circ), 27(\Delta)$.

measure for each figure. Equation (7) can be used directly in (5) yielding

$$
\begin{aligned}
h_{i} & \approx \int_{z_{1}=(i-0.5) d}^{z_{2}=(i+0.5) d} f_{\mathrm{TN}}\left(z, \mu, \sigma,-\lambda_{d}\right) d z \\
& =\frac{\operatorname{erf}\left(\left(z_{2}-\mu\right) / \sqrt{2} \sigma\right)-\operatorname{erf}\left(\left(z_{1}-\mu\right) / \sqrt{2} \sigma\right)}{1-\operatorname{erf}\left(\left(-\lambda_{d}-\mu\right) / \sqrt{2} \sigma\right)}
\end{aligned}
$$

where $\operatorname{erf}(\cdot)$ is the error function [25, Equation 7.1.1.].

We now seek a model for the parameters of the truncated normal distribution. There are two unknown parameters, $\mu$ and $\sigma$, in the truncated normal distribution, and we have two variable signal parameters, on which the shape of the distribution of $z$ has been seen to depend, namely, $\lambda_{d}$ and $\lambda_{t}$, or, equivalently, the design point, $C / N_{0}^{D}$, and the mismatch, $a$, between $C / N_{0}^{T}$ and $C / N_{0}^{D}$. Thus, we seek expressions for $\mu$ and $\sigma$, as functions of the signal parameters, in the range typically encountered for GPS acquisition, that is, $20 \mathrm{~dB}-\mathrm{Hz}$ $\leq C / N_{0}^{D} \leq 50 \mathrm{~dB}-\mathrm{Hz}$, and attenuation of $-10 \mathrm{~dB} \leq a \leq 2 \mathrm{~dB}$. A least-squares fit was carried out across this range of signal parameters, and some of the resultant $\mu$ and $\sigma$ values are illustrated in Figures 4 and 5. Parameters for both $H_{1}$ and $H_{0}$ cells are illustrated. It should be noted that, in an $H_{0}$ cell, the distribution is independent of the mismatch term, $a$, (since the signal is not present or not correlated).

The following observations can be made from Figures 4 and 5: both $\mu$ and $\sigma$ are "well-behaved" functions of $C / N_{0}^{D}$ and $a$, in the sense that the curves are continuous and not oscillatory. The mean, $\mu$, is strongly dependent on both $C / N_{0}^{D}$ and $a$, in particular, when $C / N_{0}^{D}$ is large $\left(C / N_{0}^{D} \geq 35 \mathrm{~dB}-\mathrm{Hz}\right)$. The standard deviation, $\sigma$, is strongly dependent on $C / N_{0}^{D}$ and very weakly dependent on $a$. The inherent smoothness of the curves in the $C / N_{0}^{D}$ and $a$ dimensions suggests that a suitable approach to obtaining expressions for the functions, $\mu=\mu\left(C / N_{0}^{D}, a\right)$ and $\sigma=\sigma\left(C / N_{0}^{D}, a\right)$, is a Taylor series expansion about the centre point, $\left\{\Psi_{c}, a_{c}\right\}$, of the $\left\{C / N_{0}^{D}, a\right\}$ space. The $H_{1}$ and $H_{0}$ cases are treated separately to improve the accuracy of the model in each case.

Using the notation $\Psi \equiv C / N_{0}^{D}$, for notational simplicity only, the Taylor series expansion for the mean of the distribution in an $H_{1}$ cell, $\mu_{1}$, is written as:

$$
\begin{aligned}
& \mu_{1}(\Psi, a) \\
& =\mu_{1}\left(\Psi_{c}, a_{c}\right) \\
& \quad+\left.\sum_{j=1}^{\infty} \frac{1}{j !}\left[\left(\Psi-\Psi_{c}\right) \frac{\partial}{\partial \Psi}+\left(a-a_{c}\right) \frac{\partial}{\partial a}\right]^{j} \mu_{1}(\Psi, a)\right|_{\left(\Psi_{c}, a_{c}\right)} \\
& \quad \approx \mu_{1}\left(\Psi_{c}, a_{c}\right)+\sum_{j=1}^{\infty} \sum_{i=0}^{j} \gamma_{j, i}^{\mu_{1}}\left(\Psi-\Psi_{c}\right)^{i}\left(a-a_{c}\right)^{j-i},
\end{aligned}
$$

where the notation for the Taylor series expansions should be interpreted as follows (e.g., $j=2$ ):

$$
\begin{aligned}
& {\left[\left(\Psi-\Psi_{c}\right) \frac{\partial}{\partial \Psi}+\left(a-a_{c}\right) \frac{\partial}{\partial a}\right]^{2} \mu(\Psi, a)} \\
& =\left(\Psi-\Psi_{c}\right)^{2} \frac{\partial^{2} \mu(\Psi, a)}{\partial \Psi^{2}}+2\left(\Psi-\Psi_{c}\right)\left(a-a_{c}\right) \frac{\partial^{2} \mu(\Psi, a)}{\partial \Psi \partial a} \\
& \quad+\left(a-a_{c}\right)^{2} \frac{\partial^{2} \mu(\Psi, a)}{\partial a^{2}},
\end{aligned}
$$




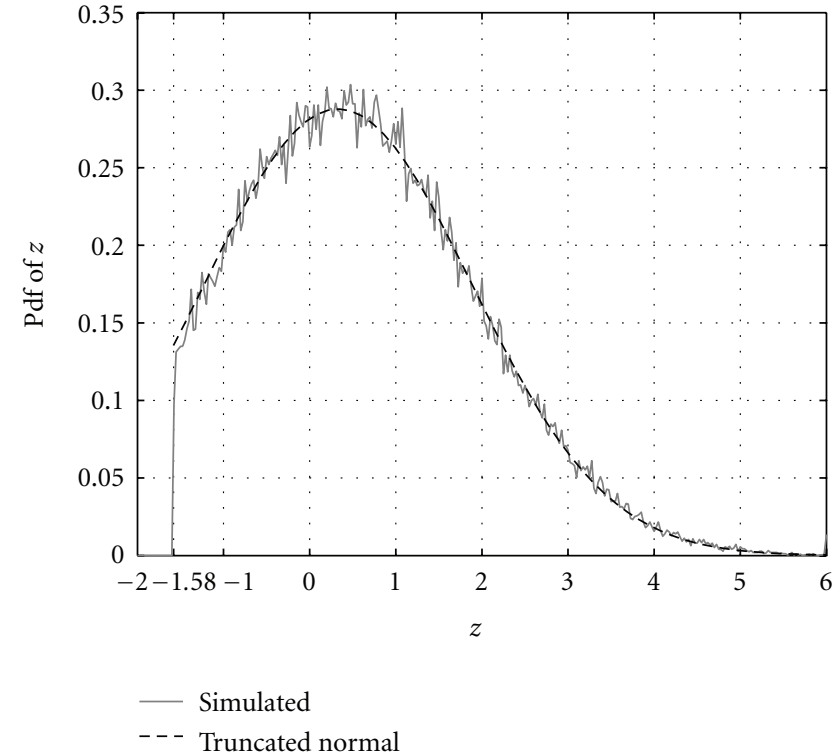

(a) $a=0 \mathrm{~dB}$

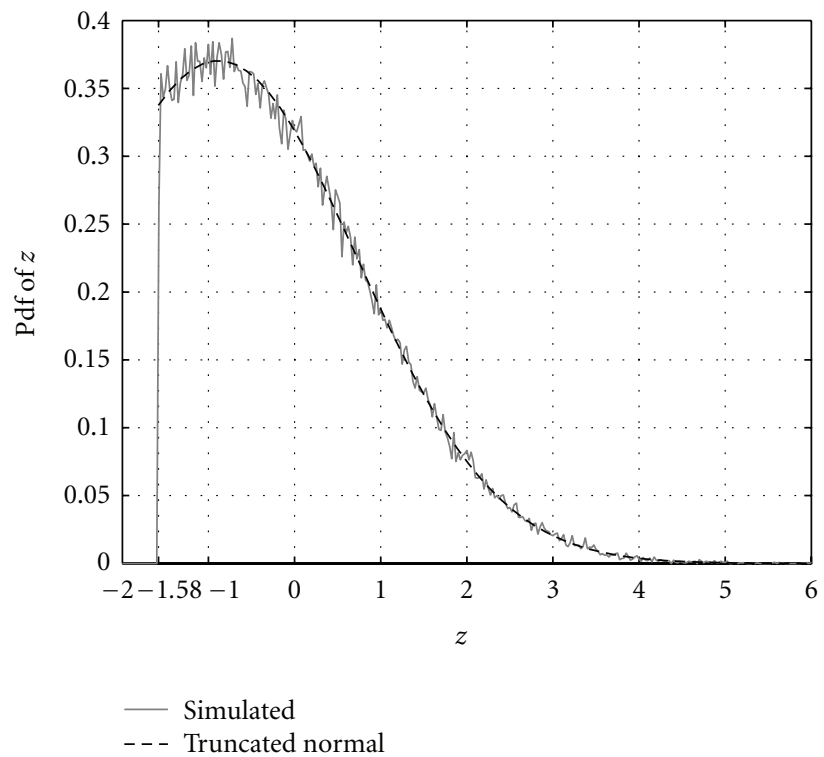

(b) $a=-4 \mathrm{~dB}$

Figure 2: Pdfs of $z$ for $C / N_{0}^{D}=32 \mathrm{~dB}-\mathrm{Hz}$ : simulated results and truncated normal approximation $\left(\lambda_{d}=1.58\right)$.

and where $\gamma_{j, i}^{\mu_{1}}$ are the series coefficients, which will be determined by a least-squares fit using the previously evaluated numerical results for $\mu_{1}\left(C / N_{0}^{D}, a\right)$. Similarly, the Taylor series expansion for the standard deviation of the distribution in an $H_{1}$ cell, $\sigma_{1}$, is given by:

$$
\begin{aligned}
& \sigma_{1}(\Psi, a) \\
& =\sigma_{1}\left(\Psi_{c}, a_{c}\right) \\
& \quad+\left.\sum_{j=1}^{\infty} \frac{1}{j !}\left[\left(\Psi-\Psi_{c}\right) \frac{\partial}{\partial \Psi}+\left(a-a_{c}\right) \frac{\partial}{\partial a}\right]^{j} \sigma_{1}(\Psi, a)\right|_{\left(\Psi_{c}, a_{c}\right)} \\
& \quad \approx \sigma_{1}\left(\Psi_{c}, a_{c}\right)+\sum_{j=1}^{\infty} \sum_{i=0}^{j} \gamma_{j, i}^{\sigma_{1}}\left(\Psi-\Psi_{c}\right)^{i}\left(a-a_{c}\right)^{j-i},
\end{aligned}
$$

with series coefficients, $\gamma_{j, i}^{\sigma_{1}}$. In an $H_{0}$ cell, we have seen that the results are independent of $a$, so the series expansions reduce to

$$
\begin{aligned}
& \mu_{0}(\Psi) \approx \mu_{0}\left(\Psi_{c}\right)+\sum_{j=1}^{\infty} \gamma_{j}^{\mu_{0}}\left(\Psi-\Psi_{c}\right)^{j}, \\
& \sigma_{0}(\Psi) \approx \sigma_{0}\left(\Psi_{c}\right)+\sum_{j=1}^{\infty} \gamma_{j}^{\sigma_{0}}\left(\Psi-\Psi_{c}\right)^{j} .
\end{aligned}
$$

The infinite sums in (9)-(12) are, of course, not suitable for practical models of the parameters, so each series must be truncated to a suitable maximum order; here, the maximum order for each model was determined by analysing various goodness-of-fit measures, such as Akaike's Information Criterion (AIC) [26] and the Bayes Information Criterion (BIC) [27]. These measures are attractive for the
TABLE 1: Mean percentage error between numerical and modeled parameters.

\begin{tabular}{ccccc}
\hline & $\mu_{0}$ & $\sigma_{0}$ & $\mu_{1}$ & $\sigma_{1}$ \\
\hline Mean error [\%] & 0.00718 & 0.00982 & 0.0218 & 0.00637 \\
\hline
\end{tabular}

type of modeling problem considered here, as they penalise the addition of extra terms in the model to avoid the choice of a trivial model, with an apparently perfect fit, achieved when there is a unique term for each data point available. The order of the model, for each parameter, was chosen as the order beyond which the incremental improvement in fit, using the AIC, was $\leq 1 \%$ (i.e., further increasing the order of the model resulted in a decrease of less than $1 \%$ in the AIC); the resultant orders for the models are as follows: $J_{\mu_{0}}=13$, $J_{\sigma_{0}}=8, J_{\mu_{1}}=21$, and $J_{\sigma_{1}}=22$.

The values of the coefficients, $\gamma_{j, i}^{\mu_{1}}, \gamma_{j, i}^{\sigma_{1}}, \gamma_{j}^{\sigma_{0}}$, and $\gamma_{j}^{\mu_{0}}$, were determined using a nonlinear least-squares fit, for numerically evaluated data in the range, $20 \mathrm{~dB}-\mathrm{Hz} \leq \mathrm{C} / \mathrm{N}_{0}^{D} \leq$ $50 \mathrm{~dB}-\mathrm{Hz},-10 \mathrm{~dB} \leq a \leq 2 \mathrm{~dB}$, with step size of $0.5 \mathrm{~dB}$ in each dimension and centre point, $\Psi_{c}=35 \mathrm{~dB}-\mathrm{Hz}, a_{c}=-4 \mathrm{~dB}$; the results are listed in Appendix B. The mean percentage error between the numerical parameters and the Taylor series models, for each case, are listed in Table 1 and can be seen to be negligible.

\section{Validation of the New Approximation}

With a closed form representation for the $h_{i}$ probabilities now available, (8), the methods of either Proakis or Wald and Girshick can be used to accurately evaluate the ASN and OCF of the SPRT for GPS acquisition. Here, we present results comparing the theoretical performance of the 


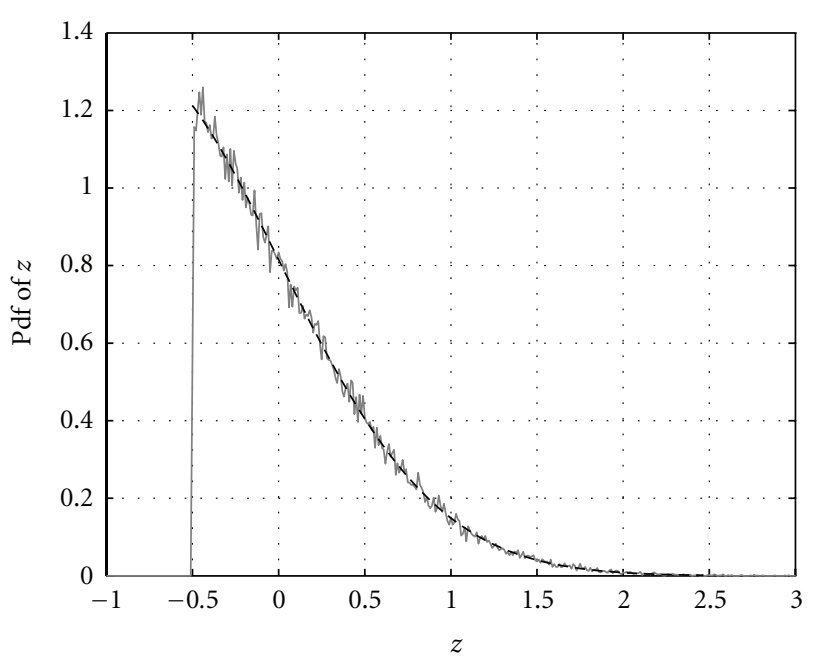

- Simulated

- - - Truncated normal

(a) $a=0 \mathrm{~dB}$

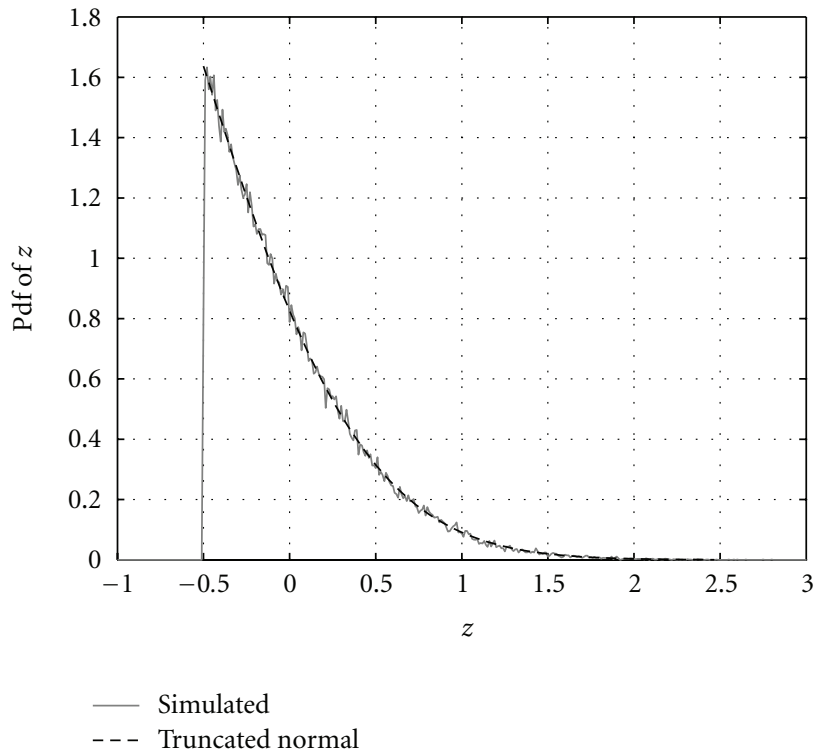

(b) $a=-4 \mathrm{~dB}$

Figure 3: Pdfs of $z$ for $C / N_{0}^{D}=27 \mathrm{~dB}-\mathrm{Hz}$ : simulated results and truncated normal approximation $\left(\lambda_{d}=0.5\right)$.

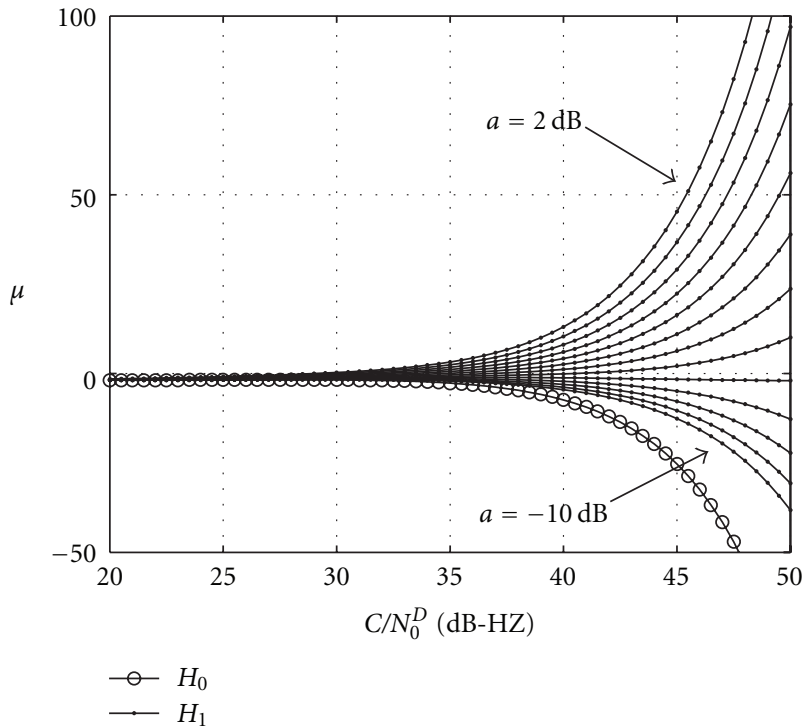

FIGURE 4: Least-squares estimates of truncated normal parameter, $\mu$, versus design point carrier-to-noise ratio, $C / N_{0}^{D}$, with varying attenuation (in steps of $1 \mathrm{~dB}$ from $a=2 \mathrm{~dB}$ to $a=-10 \mathrm{~dB}$ ).

SPRT, based on the truncated normal approximation, with results from Monte Carlo simulations (carried out under the same signal generation conditions as outlined in Section 3). The theoretical results are calculated using Proakis' aforementioned Markov chain method.

Figure 6 shows the mean dwell time in an $H_{1}$ cell, normalised by its simulated value at $a=0 \mathrm{~dB}$ for each $C / N_{0}^{D}$ value (listed in the legend), with a target performance of $\left\{P_{d c}^{(T)}=0.98, P_{f a c}^{(T)}=10^{-4}\right\}$. The probability of detection,

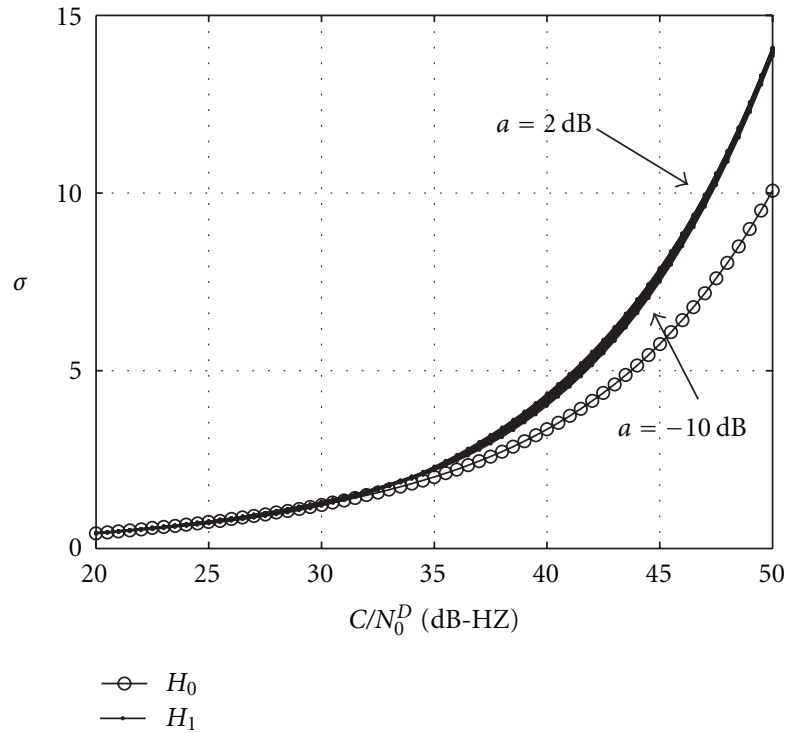

FIGURE 5: Least-squares estimates of truncated normal parameter, $\sigma$, versus design point carrier-to-noise ratio, $C / N_{0}^{D}$, with varying attenuation (in steps of $1 \mathrm{~dB}$ from $a=2 \mathrm{~dB}$ to $a=-10 \mathrm{~dB}$ ).

$P_{d c}$, is shown in Figure 7. It should be noted that Wald's small signal approximation for $P_{d c}$ takes account only of the relative values of $C / N_{0}^{D}$ and $C / N_{0}^{T}$ and not their absolute values; hence, it operates on a "one curve fits all" basis, which is highly inaccurate for medium weak to strong signals $\left(C / N_{0}^{D}>24 \mathrm{~dB}-\mathrm{Hz}\right)$. It is clear that the new truncated normal approximation results in significantly more accurate performance analysis than Wald's small signal approximations, especially when $C / N_{0}^{D}$ is large. Similar accuracy was achieved for the $H_{0}$ performance. For $C / N_{0}^{D}=24 \mathrm{~dB}-\mathrm{Hz}$, there is 


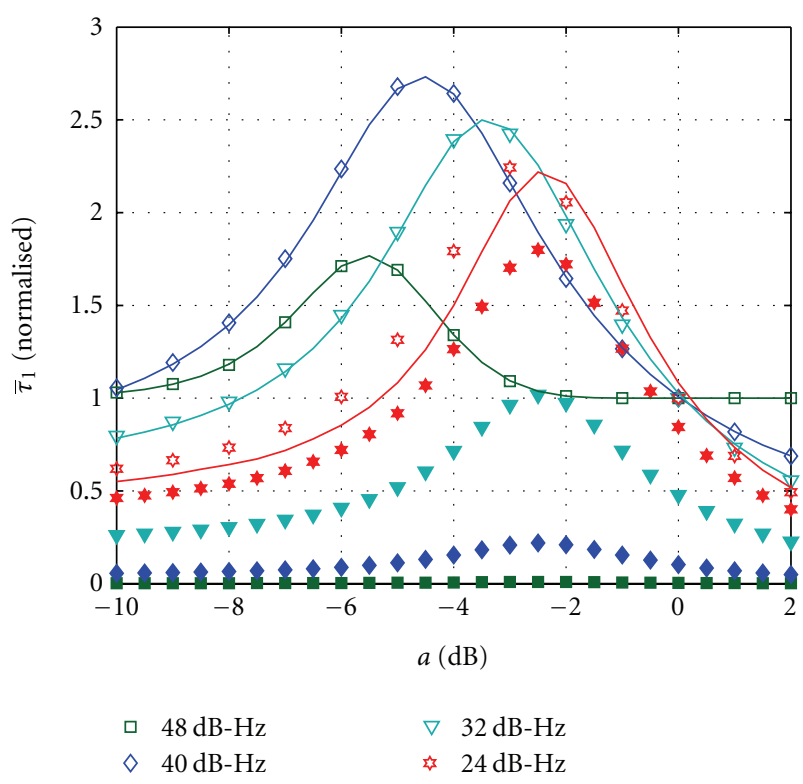

Figure 6: Normalised SPRT dwell time in an $H_{1}$ cell versus attenuation: simulation (hollow markers), new approximation (solid lines), and Wald approximation (filled markers).

a small discrepancy between the new approximation and the simulated values. This is due to the approximation of the continuous distribution of $z$ by a discrete distribution. The effect is only pronounced for very weak signals as the step size, $d$, must become extremely small for accurate results and the corresponding matrices which must be manipulated to determine the performance metrics become unmanageably large with the available computational resources.

The final validation of the usefulness of our new approximation is to compare the computational resources required for its calculation to that of previously available methods. We have mentioned that the exact calculation of the ASN and OCF for the SPRT, using either Wald and Girshick's or Proakis' method, involves the evaluation of the probabilities, $h_{i}=\operatorname{Pr}(z=i d)$. The aim of our approximation was to simplify the accurate evaluation of these $h_{i}$ values. Thus, to compare the efficiency improvement for SPRT performance analysis of our new approximation over the existing methods for evaluating the ASN and OCF, we simply evaluate the mean CPU time required for the evaluation of the $h_{i}$ values. The following methods are included in the comparison:

(1) $h_{i}$ values approximated using numerical integration of (A.15) with the $h_{x_{i}}$ values based on (A.5) and a numerical solution for $x=x(z)$,

(2) $h_{i}$ values approximated using (8).

The CPU time samples were generated in Mathematica, due to the availability, in this software package, of specialised methods for calculating Bessel functions, error functions, and so forth. The reason for using this commercial software package was to provide the fairest comparison possible with the available resources, one which is unbiased by inefficiencies in the evaluation of the special mathematical functions,

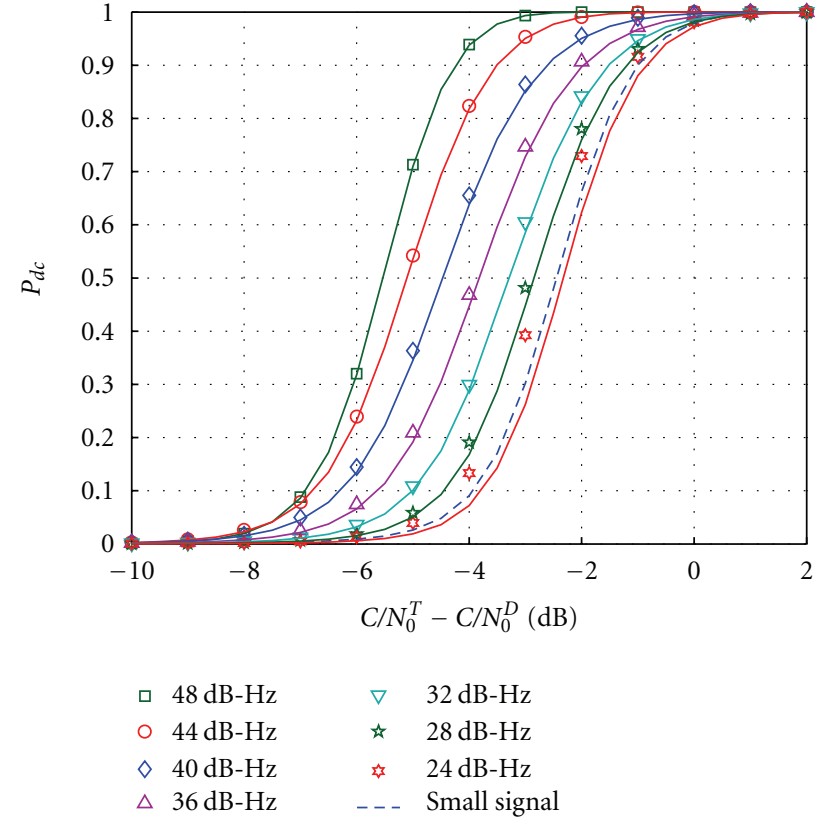

FIGURE 7: SPRT detection probability versus attenuation: simulation (hollow markers), truncated normal approximation (solid lines), and Wald's small signal approximation (dashed line).

TABLE 2: Mean CPU time ratio $\bar{T}_{2} / \bar{T}_{1} \times 100(\%)$.

\begin{tabular}{lccc}
\hline $\mathrm{C} / \mathrm{N}_{0}^{D}$ & & $a(\mathrm{~dB})$ & \\
$(\mathrm{dB}-\mathrm{Hz})$ & 0 & -4 & -8 \\
\hline 44 & 0.8789 & 0.8241 & 0.8238 \\
40 & 0.7757 & 0.7706 & 0.7636 \\
36 & 0.8588 & 0.8611 & 0.8856 \\
32 & 0.8447 & 0.8605 & 0.8562 \\
28 & 0.9630 & 1.0568 & 1.0304 \\
\hline
\end{tabular}

such as the Bessel functions in Method 1 and the error functions in Method 2. For each pair of $\left\{C / N_{0}^{D}, a\right\}$, values whose performance was computed, 100 sample CPU times were measured using the total time taken, in seconds, for the CPU to evaluate $h_{i}, i=\left\lfloor z_{\min } / d\right\rfloor, \ldots,\left\lceil z_{\max } / d\right\rceil$. It should be noted that the system caches were cleared between samples to ensure a "worst case" time estimate. Table 2 reports the ratio of $\bar{T}_{2}$ to $\bar{T}_{1}$, where $\bar{T}_{1}$ is the mean CPU time for Method 1 (the numerical method) and $\bar{T}_{2}$ is the mean CPU time for Method 2 (the truncated normal approximation), expressed as a percentage. It is clear that the truncated normal approximation provides a very significant improvement over the old numerical method, requiring approximately $1 \%$ or less of the numerical method's CPU time in all cases tested. The comparison was carried out on both a Windows and a Unix platform, with a similar time saving observed on each platform.

It is noted, here, that the suitability of the truncated normal approximation and the resultant new expressions for the SPRT metrics have been derived through observation of the shape of the pdf followed by a numerical curvefitting technique, and not through a rigorous mathematical 
or probabilistic approach. However, in the absence of an analytical or semianalytical solution, the numerical curvefitting approach is a practical solution to the problem, which works well in practice. The improved accuracy of the performance analysis justifies the use of this solution. Functional forms for $\mu=\mu\left(\lambda_{d}, \lambda_{t}\right)$ and $\sigma=\sigma\left(\lambda_{d}, \lambda_{t}\right)$ might, perhaps, be attainable by equating $f_{z}\left(z, \lambda_{d}, \lambda_{t}\right)$ and $f_{\mathrm{TN}}\left(z, \mu, \sigma,-\lambda_{d}\right)$ and carrying out lengthy manipulation of the equations. However, initial attempts have shown that such a procedure appears to be very complicated, and the added benefits of functional forms over the parameter approximations given above, (9)-(12), are not expected by the authors to be sufficiently advantageous to justify such an effort.

\section{Real Signal Verification}

Here, the accuracy of the derived theoretical analysis for the SPRT is further verified by comparison with simulated results and with results from tests on signals collected from live GPS L1 C/A satellites. The data collection was carried out in a location at the University of Calgary, Canada, where there is a clear view of the sky in all directions, and multipath effects are known to be low. Two datasets were collected with the $C / N_{0}$ levels of the received signals varied by means of a variable attenuator to simulate low $C / N_{0}$ conditions in a controlled manner. Dataset 1 consisted of five epochs of ten minutes each, with fixed nominal attenuation levels of 0 , $-10,-15,-20$, and $-26 \mathrm{~dB}$. Dataset 2 was collected over a period of six minutes with zero attenuation for the first two minutes, and the attenuation decreased by $1 \mathrm{~dB}$ every four seconds thereafter. There were multiple satellites visible during collection of each dataset, and their received $C / N_{0}$ values varied according to their elevation angles. Thus, there was a wide range of signal strengths present in the collected data.

The known signal strength condition was tested using Dataset 1. The SPRT acquisition process was carried out for an unused PRN code (PRN 36) in order to measure the $H_{0}$ cell statistics, and estimated parameters, obtained from the University of Calgary's GSNRx software receiver [28], were used to estimate the $H_{1}$ cell statistics. The range of signal strengths available in Dataset 1 allowed a good distribution of $C / N_{0}$ levels to be considered. Figure 8 shows the simulated, theoretical, and real signal measurements for $\bar{\tau}_{0}$ and $\bar{\tau}_{1}$, showing excellent agreement between the three sets of results and, hence, validating the simulated, and theoretical results. The $H_{0}$ results agree within \pm 0.2 standard deviations of the theoretical values, with the $H_{1}$ results falling within less than one standard deviation of the equivalent theoretical results.

The effect of attenuation was investigated using signals from Dataset 2. Repeated acquisitions were carried out on one of the visible satellites, PRN 31, for a fixed design point, $C / N_{0}^{D}=44 \mathrm{~dB}-\mathrm{Hz}$, with $C / N_{0}^{T}$ varying according to the known attenuation level, as described above. The same process was carried out for another satellite which had a lower received signal power, PRN 20, with $C / N_{0}^{D}=36 \mathrm{~dB}$ $\mathrm{Hz}$. The results are illustrated in Figure 9, showing excellent agreement between theoretical, simulated and real signal

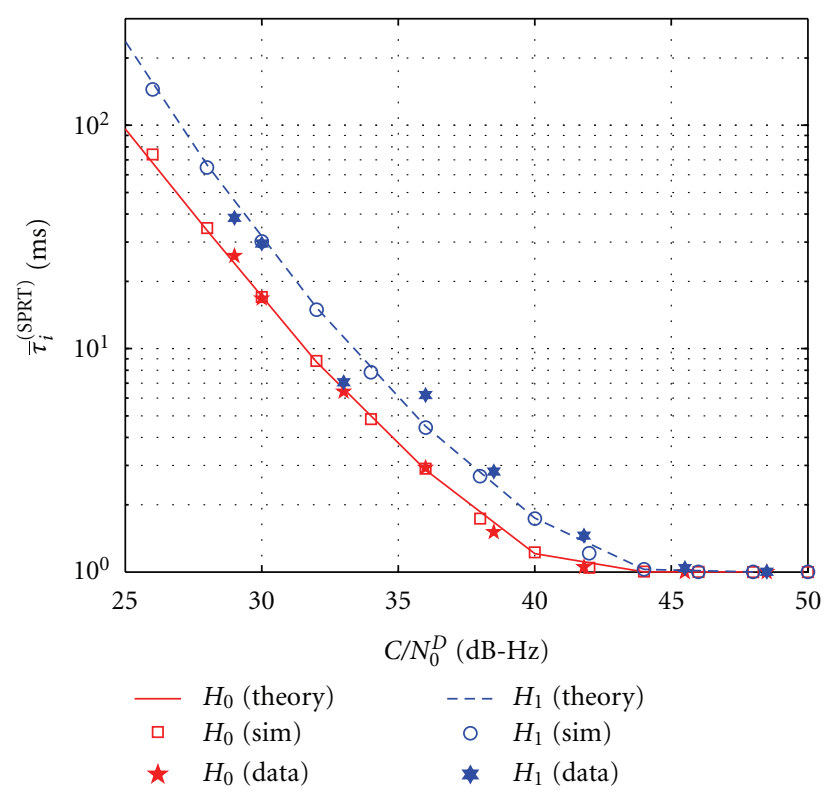

Figure 8: SPRT mean dwell time versus $C / N_{0}$ : simulated, theoretical, and real signal results.

results for the $C / N_{0}^{D}=44 \mathrm{~dB}-\mathrm{Hz}$ case. The variation in $\bar{\tau}_{1}$, in the case of $C / N_{0}^{D}=36 \mathrm{~dB}-\mathrm{Hz}$, is due to the fact that the average was taken over approximately 100-150 samples for each data point, compared to approximately 1500 samples for the $44 \mathrm{~dB}-\mathrm{Hz}$ case. The limit on the number of samples is, of course, due to the length of the collected data and the dwell time requirements.

\section{Comparison with Suboptimal Strategies}

Returning to the motivation of the work, the goal was to provide an accurate performance analysis for the SPRT to allow comparison between it and suboptimal decisionmaking strategies to be carried out at the design stage. The fundamental trade-off between performance and implementation is an important compromise for receiver design and it is considered, by the authors, to be vital that the optimally achievable performance is accurately known, as a benchmark against which other strategies should be compared. Now that an accurate and efficient theoretical analysis has been derived for the SPRT in the typical GPS $C / N_{0}$ range, its performance can be readily compared to that of other acquisition strategies for receiver design purposes.

A simulation-based comparison between the SPRT and the SDD was reported by the authors in [10], illustrating that there exists a $C / N_{0}$ range for which even the worst case mean dwell time for the SPRT is less than the fixed SDD dwell time. Here, a theoretical comparison between the SPRT and two suboptimal up-down counter (UDC) strategies, namely, the classical Tong detector (or singlethreshold UDC) [12] and a dual-threshold UDC strategy [13], is presented, illustrating a use of the derived theoretical approximation for the SPRT. The effect of mismatch of the 


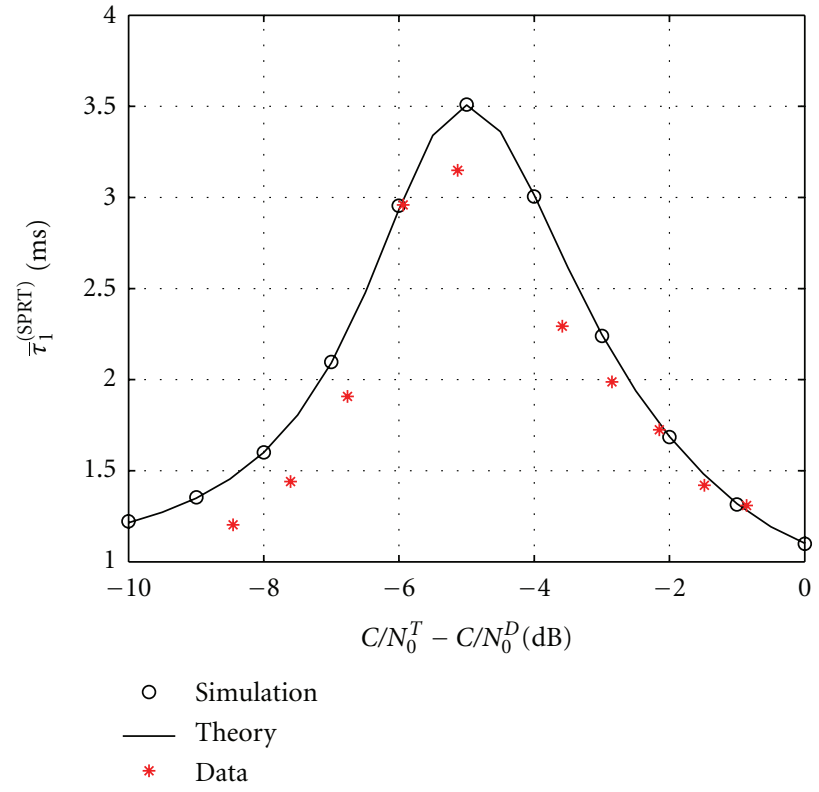

(a) $C / N_{0}^{D}=44 \mathrm{~dB}-\mathrm{Hz}(\mathrm{PRN} 31)$

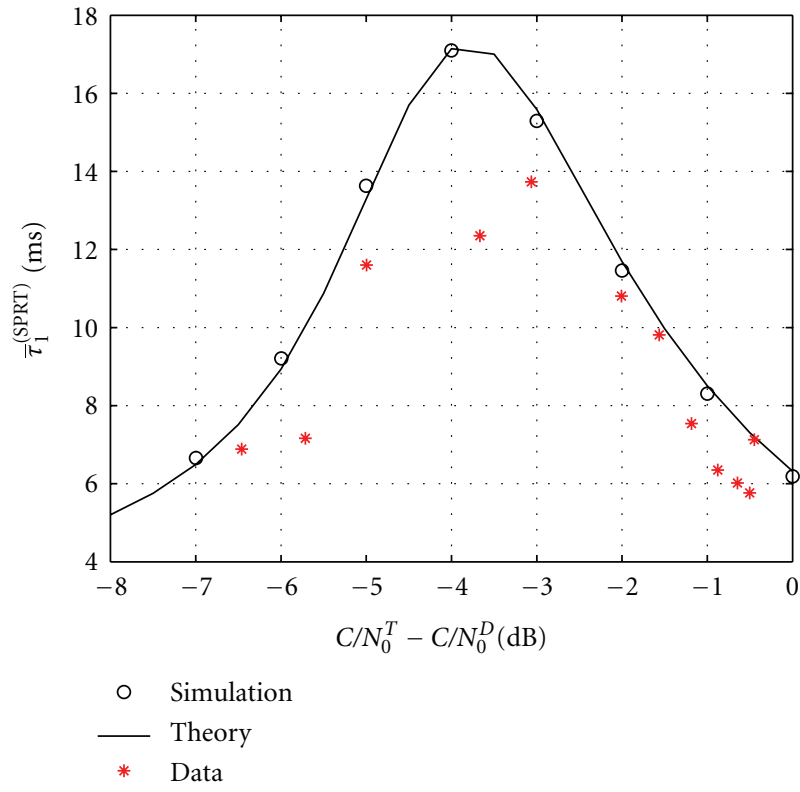

(b) $C / N_{0}^{D}=36 \mathrm{~dB}-\mathrm{Hz}(\mathrm{PRN} 20)$

FIGURE 9: SPRT mean dwell time versus attenuation: simulated, theoretical, and real signal results.

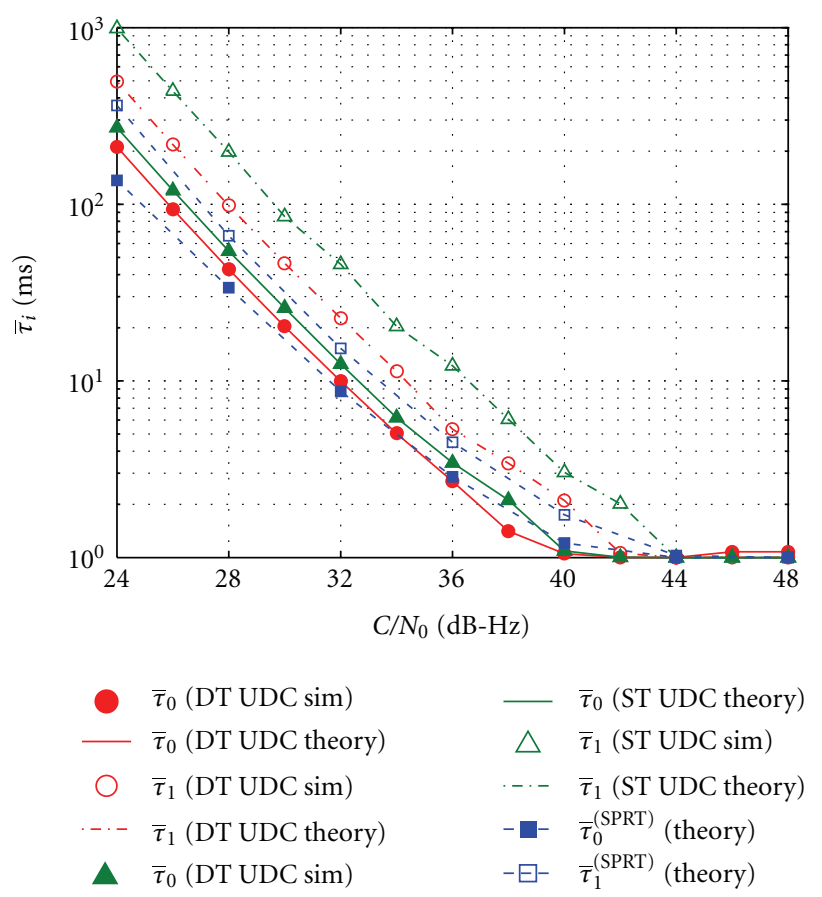

FIGURE 10: Dwell time versus carrier-to-noise ratio; $\left\{P_{d c}^{(T)}=0.98\right.$, $\left.P_{f a c}^{(T)}=10^{-4}\right\}$.

received $C / N_{0}$ relative to the design point is considered for each strategy in the comparison.

Figure 10 shows the mean $H_{1}$ and $H_{0}$ cell dwell times for a single-threshold UDC (STUDC), a dual-threshold UDC (DTUDC), and the SPRT, under the condition that $C / N_{0}^{D}=$ $C / N_{0}^{T}$. For the UDC strategies, the value of $C / N_{0}^{D}$ and the desired probabilities of detection and false alarm determine the optimal dwell parameters. Both theoretical and simulated

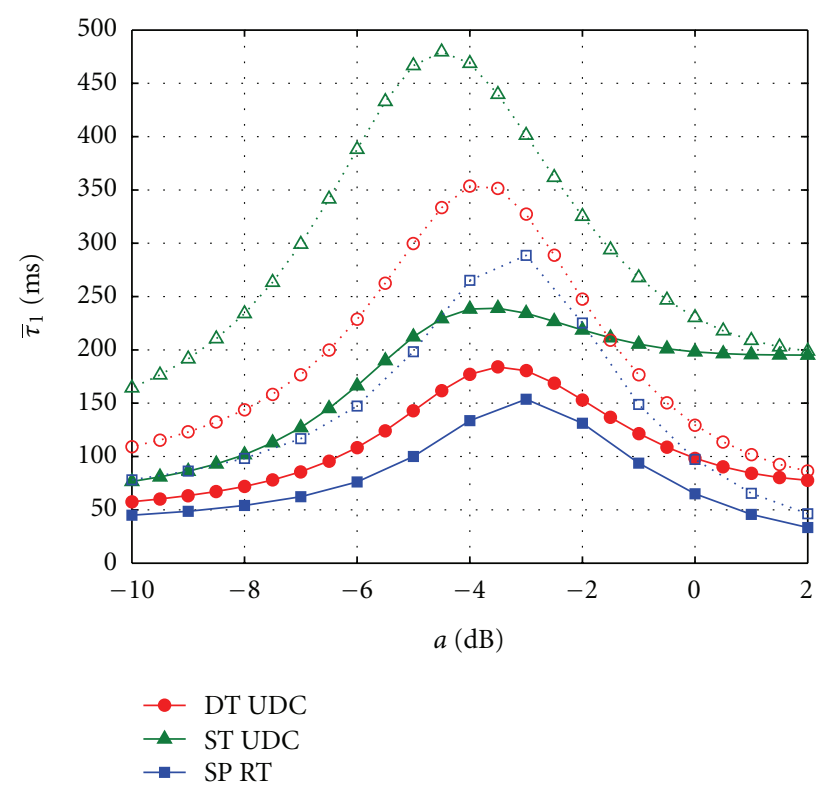

Figure 11: Comparison of mean and variance of dwell time for DTUDC, STUDC, and SPRT: $\bar{\tau}_{1}$ (solid lines, filled markers), $\bar{\tau}_{1}+$ $\sqrt{\operatorname{Var}\left[\tau_{1}\right]}$ (dashed lines, hollow markers); $C / N_{0}^{D}=28 \mathrm{dBHz},\left\{P_{d c}^{(T)}=\right.$ $\left.0.98, P_{\text {fac }}^{(T)}=10^{-4}\right\}$.

results are shown for the UDC strategies as these have not been previously verified in this work. It is clear that, in the case when the received and design point $C / N_{0}$ values are equal, the SPRT, as expected, outperforms the suboptimal strategies.

Considering the case of unknown power levels, Figure 11 shows the mean $H_{1}$ cell dwell time versus attenuation for each strategy. Also illustrated in the figure is the mean plus 
one standard deviation dwell time which provides a measure of how dwell time variance affects the performance. In this case, it is clear that the SPRT significantly outperforms both suboptimal strategies across the entire range of attenuation. Such theoretically derived performance predictions can be used by receiver designers in analysing the trade-off between performance and computational load in the strategy choice.

\section{Conclusions}

In this work, a new approximation for the distribution of the probability ratio for the SPRT with noncoherent correlation was presented. The main advantage of this approximation is that it provides the distribution of the probability ratio in a simplified form which may be easily manipulated to provide the performance metrics of the SPRT. Approximate expressions for the parameters of the new distribution were provided and shown to yield highly accurate results for the performance of the SPRT. Furthermore, for the cases considered here, the computation time required for the new approximation was shown to be less than $1 \%$ of that required for numerical methods with similar accuracy, validating the usefulness of the approximation.

Using the new approximation, the theoretical performance of the SPRT was compared to that of suboptimal updown counter strategies for a wide range of design points and for varying attenuation levels, and it was shown that the SPRT provides a superior mean dwell time performance in the GPS $C / N_{0}$ range of interest.

\section{Appendices}

\section{A. Manipulation of Known Expressions for Probability Ratio Distribution}

Here, we manipulate known expressions involving the correlator output, $x$, and the probability ratio, $z$, in an attempt to find an integrable form for the distribution of $z$. Relating the distributions of the correlator output and the probability ratio by

$$
\int f_{z(x)}\left(z(x), \lambda_{d}, \lambda_{t}\right) d z(x)=\int f_{x}\left(x, \lambda_{t}\right) d x,
$$

and differentiating, we have

$$
f_{z}\left(x, \lambda_{d}, \lambda_{t}\right) \frac{d z(x)}{d x}=f_{x}\left(x, \lambda_{t}\right)
$$

From (3), we have $z(x)=-\lambda_{d}+\ln \left(I_{0}\left(2 \sqrt{x \lambda_{d}}\right)\right)$, which can be differentiated to give

$$
\frac{d z(x)}{d x}=\sqrt{\frac{\lambda_{d}}{x}}\left(\frac{I_{1}\left(2 \sqrt{x \lambda_{d}}\right)}{I_{0}\left(2 \sqrt{x \lambda_{d}}\right)}\right) .
$$

By combining [29, Equations 2.3-21 and 2.3-29], we can write the pdf of the normalised NCCD correlator output (with $K=1$ ) as

$$
f_{x}\left(x, \lambda_{t}\right)=\exp \left(-x-\lambda_{t}\right) I_{0}\left(2 \sqrt{x \lambda_{t}}\right)
$$

TABLE 3: Regions of convergence and maximum correlator output value.

\begin{tabular}{lcc}
\hline$C / N_{0}^{D}(\mathrm{~dB}-\mathrm{Hz})$ & $\lim _{k \rightarrow \infty}\left|e_{k}\right| /\left|e_{k+1}\right|$ & $x_{\max }$ \\
\hline 39 & 0.4632 & 58.0293 \\
37 & 0.7341 & 45.1286 \\
35 & 1.1635 & 36.0558 \\
33 & 1.8440 & 29.8072 \\
31 & 2.9225 & 25.8072 \\
29 & 4.6319 & 23.1259 \\
27 & 7.3410 & 21.3224 \\
25 & 11.635 & 19.8665 \\
23 & 18.440 & 18.6131 \\
21 & 29.225 & 18.473 \\
\hline
\end{tabular}

where $\lambda_{t}=0$ in an $H_{0}$ cell and $\lambda_{t}>0$ in an $H_{1}$ cell. Substituting (A.3) and (A.4) into (A.2) and rearranging yields the following expression for the pdf of the probability ratio, $z$, in terms of the correlator output, $x$ :

$$
\begin{aligned}
& f_{z(x)}\left(x, \lambda_{d}, \lambda_{t}\right) \\
& \quad=\exp \left(-x-\lambda_{t}\right) I_{0}\left(2 \sqrt{x \lambda_{t}}\right) \sqrt{\frac{x}{\lambda_{d}}}\left(\frac{I_{0}\left(2 \sqrt{x \lambda_{d}}\right)}{I_{1}\left(2 \sqrt{x \lambda_{d}}\right)}\right) .
\end{aligned}
$$

Due to the change of variables, the expression in (A.5) is not in a form which can be used directly in (5). If, however, an expression for the correlator output, in terms of $z$, that is, $x=x(z)$, can be found and substituted into (A.5), an expression for the pdf of the probability ratio, in terms of itself and the signal power parameters, that is, $f_{z}\left(z, \lambda_{d}, \lambda_{t}\right)$, will emerge.

Manipulation of (3) gives

$$
\exp \left(z+\lambda_{d}\right)=I_{0}\left(2 \sqrt{x \lambda_{d}}\right)
$$

both sides of which can be represented as power series, leading to the following equation, whose roots are the solution to $x=x(z)$ :

$$
\sum_{n=1}^{\infty} \frac{\left(z+\lambda_{d}\right)^{n}-\left(\left(x \lambda_{d}\right)^{n} / n !\right)}{n !}=0 .
$$

A closed form expression for $x=x(z)$ is not realisable from (A.7), to the best of the authors' knowledge. Numerical evaluation shows that the sum can be truncated at $n=N_{\max }$, without significant loss of accuracy, where the value of $N_{\max }$ depends on $\lambda_{d}$ and $\lambda_{t}$ and ranges from $N_{\max }=10$ for very weak signals, up to $N_{\max } \approx 80$, or greater, for nominal GPS signal strength (approximately $44 \mathrm{~dB}-\mathrm{Hz}[1]$ ). As such, solution of (A.7) is nontrivial.

Even if a closed form for $x=x(z)$ could be found, (A.5) is, currently, not in a form which can be readily integrated, due to the presence of the Bessel $I_{0}(\cdot)$ and $I_{1}(\cdot)$ functions, combined with the exponential term. Numerical methods could be used. Alternatively, in an effort to find an easily integrable form of (A.5) in terms of $z$, the power series 
TABLe 4: Truncated normal distribution model coefficients for $\mu_{0}$.

\begin{tabular}{lccccc}
\hline$j$ & 1 & 2 & 3 & 4 & 5 \\
\hline$\gamma_{j}^{\mu_{H_{0}}}$ & -0.406 & -0.0669 & -0.00583 & -0.000344 & 9 \\
$j$ & 6 & 7 & 8 & $1.126 e-10$ & 10 \\
$\gamma_{j}^{\mu_{H_{0}}}$ & $-6.563 e-07$ & $-3.618 e-08$ & $-7.117 e-10$ & $4.138 e-13$ \\
$j$ & 11 & 12 & 13 & \\
$\gamma_{j}^{\mu_{H_{0}}}$ & $-5.339 e-13$ & $-1.772 e-15$ & $8.061 e-16$ & \\
\hline
\end{tabular}

TABLE 5: Truncated normal distribution model coefficients for $\sigma_{0}$.

\begin{tabular}{lcccc}
\hline$j$ & 1 & 2 & 3 & 4 \\
\hline$\gamma_{j}^{\mu_{H_{0}}}$ & 0.1999 & 0.0108 & 0.000486 & $1.566 e-05$ \\
$j$ & 5 & 6 & 7 & 8 \\
$\gamma_{j}^{\mu_{H_{0}}}$ & $1.739 e-07$ & $-5.554 e-10$ & $2.719 e-10$ & $8.383 e-12$ \\
\hline
\end{tabular}

representations of the Bessel functions can be manipulated and combined as follows:

$$
\begin{aligned}
I_{0}\left(2 \sqrt{x \lambda_{d}}\right) I_{0}\left(2 \sqrt{x \lambda_{t}}\right) & =\left(\sum_{k=0}^{\infty} \frac{\left(x \lambda_{d}\right)^{k}}{k !^{2}}\right)\left(\sum_{j=0}^{\infty} \frac{\left(x \lambda_{t}\right)^{j}}{j !^{2}}\right) \\
& =\sum_{k=0}^{\infty} x^{k} c_{k},
\end{aligned}
$$

where, for $k \geq 0$,

$$
\begin{aligned}
c_{k} & =\sum_{j=0}^{k} \frac{\lambda_{d}^{j} \lambda_{t}^{k-j}}{j !^{2}(k-j) !^{2}} \\
I_{1}\left(2 \sqrt{x \lambda_{d}}\right) & =\sqrt{x \lambda_{d}}\left(\sum_{k=0}^{\infty} \frac{1}{k+1} \frac{\left(x \lambda_{d}\right)^{k}}{k !^{2}}\right) \\
& =\sqrt{x \lambda_{d}} \sum_{k=0}^{\infty} x^{k} d_{k},
\end{aligned}
$$

where, for $k \geq 0$,

$$
d_{k}=\frac{\left(\lambda_{d}\right)^{k}}{k !(k+1) !}
$$

which can be combined and simplified to yield

$$
f_{z(x)}\left(x, \lambda_{d}, \lambda_{t}\right)=\frac{\exp \left(-x-\lambda_{t}\right)}{\lambda_{d}} \sum_{k=0}^{\infty} x^{k} e_{k},
$$

where $e_{0}=c_{0} / d_{0}=1$ and, for $k>0$,

$$
e_{k}=c_{k}-\sum_{j=0}^{k-1} e_{j} d_{k-j}
$$

Using the definition of the upper incomplete Gamma function [25, Equation 6.1.1] and changing the order of integration and summation, we have

$$
\begin{aligned}
\int_{x_{1}}^{\infty} f_{z(x)}\left(x, \lambda_{d}, \lambda_{t}\right) d x & =\frac{\exp \left(\lambda_{t}\right)}{\lambda_{d}} \sum_{k=0}^{\infty} e_{k} \int_{x_{1}}^{\infty} x^{k} \exp (-x) d x \\
& =\frac{\exp \left(\lambda_{t}\right)}{\lambda_{d}} \sum_{k=0}^{\infty} e_{k} \Gamma_{k+1}\left(x_{1}\right)
\end{aligned}
$$

Then, we can define

$$
\begin{aligned}
h_{x_{i}} & \triangleq \int_{x_{l, i}}^{x_{u, i}} f_{z(x)}\left(x, \lambda_{d}, \lambda_{t}\right) d x \\
& =\frac{\exp \left(\lambda_{t}\right)}{\lambda_{d}} \sum_{k=0}^{\infty} e_{k}\left(\Gamma_{k+1}\left(x_{l, i}\right)-\Gamma_{k+1}\left(x_{u, i}\right)\right),
\end{aligned}
$$

which can be used to approximate the values of $h_{i}, i=$ $\left\lfloor z_{\min } / d\right\rfloor, \ldots,\left\lceil z_{\max } / d\right\rceil$, in $(5)$ by setting $x_{l, i}=x((i-0.5) d)$ and $x_{u, i}=x((i+0.5) d)$ and evaluating

$$
\left.h_{i} \approx h_{x_{i}} \frac{d z(x)}{d x}\right|_{x=x(i d)} .
$$

We now appear to have an expression for the $h_{i}$ probabilities, required for the evaluation of the ASN and OCF of the SPRT, using the methods of Wald and Girshick or Proakis, which does not involve the integration of Bessel functions. If the infinite sum in (A.14) can be shown to converge, such that we can truncate the sum at $k=K_{\max }$ without loss of accuracy, we will have a numerically solvable approximation for (5), albeit a nontrivial one. The region of convergence of (A.11) depends on the values of $\lambda_{d}$ and $\lambda_{t}$ through the definitions of the coefficients, $c_{k}, d_{k}$, and $e_{k}$ ). Numerical tests show that as $\lambda_{d}$ increases, the region of convergence, that is, the range of values of $x$ for which

$$
|x|<\lim _{k \rightarrow \infty} \frac{\left|e_{k}\right|}{\left|e_{k+1}\right|}
$$

is satisfied, decreases. Conversely, the range of values of $x$, over which the sum must be calculated, increases with increasing $\lambda_{t}$. Ideally, the detector will be designed such that $\lambda_{d}=\lambda_{t}$; Table 3 shows the region of convergence for a range of values of $C / N_{0}^{D}=C / N_{0}^{T}$, along with the corresponding maximum correlator value, $x_{\max }$, for which the probability in (A.14) must be calculated. The $x_{\max }$ values 


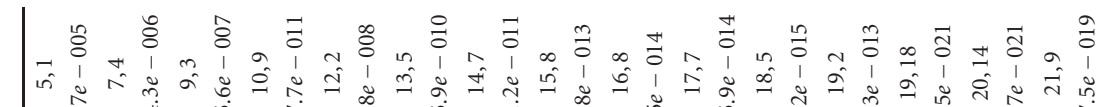

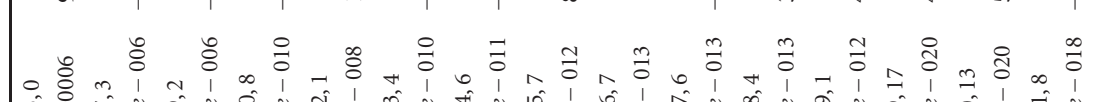

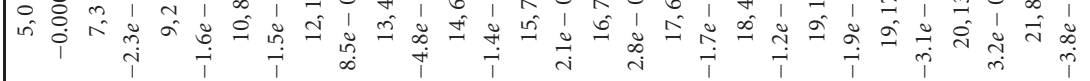

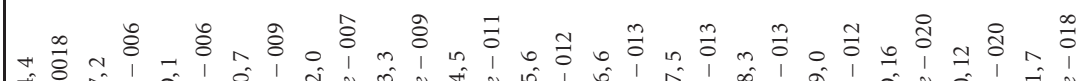

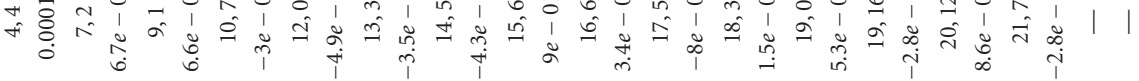

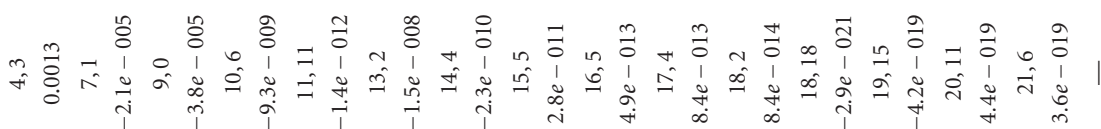

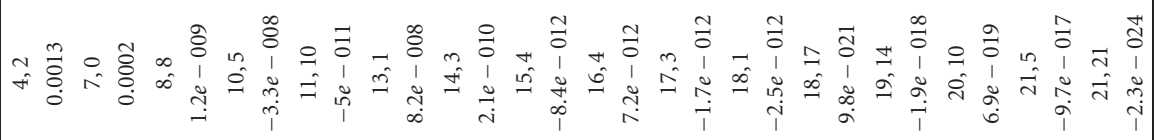

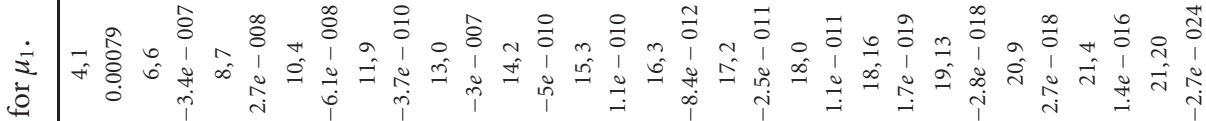

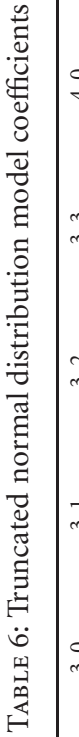

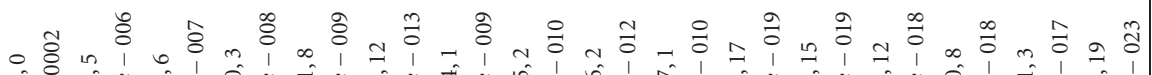

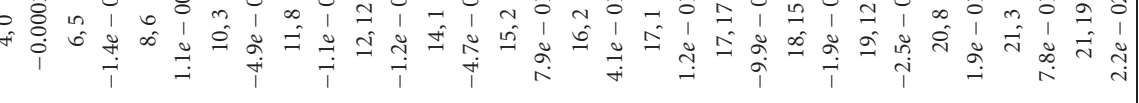

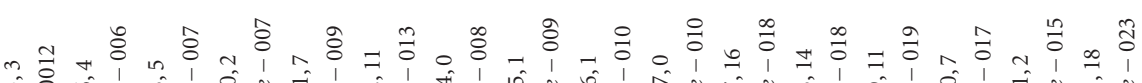

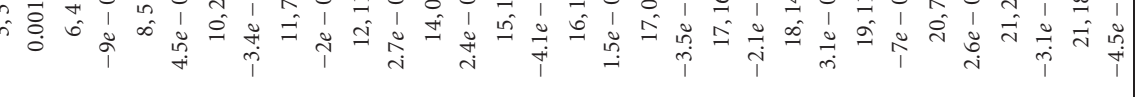

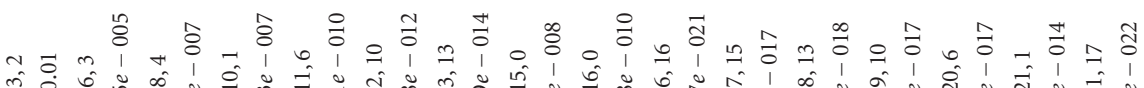

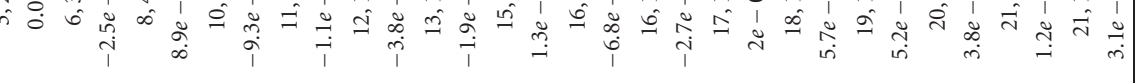

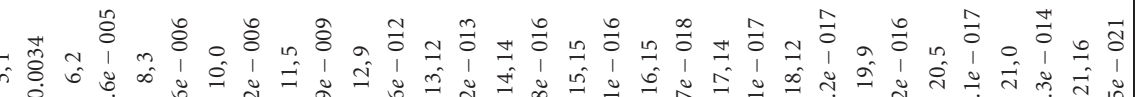

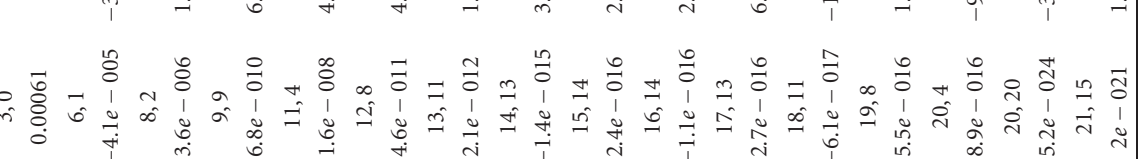

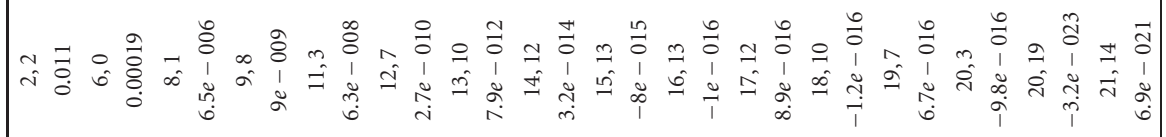

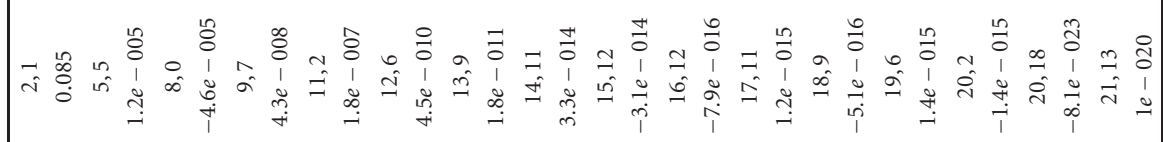

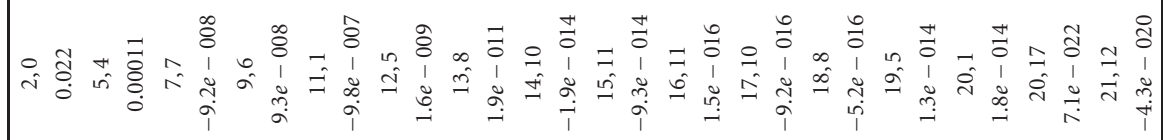

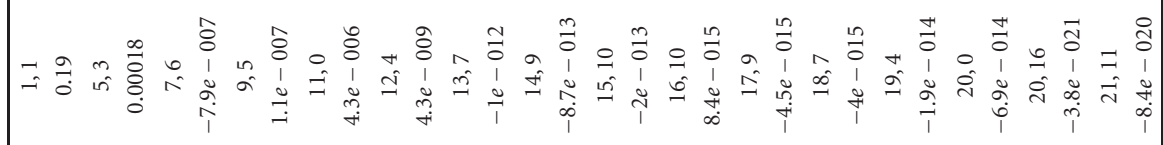

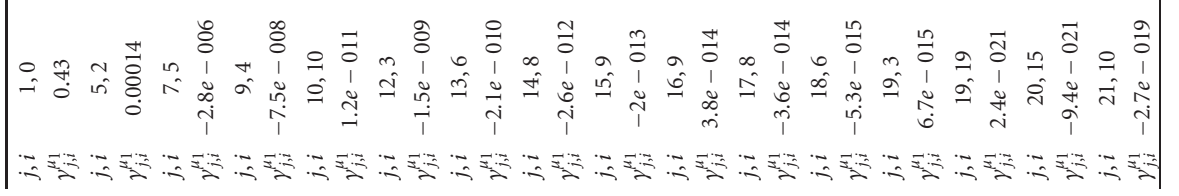




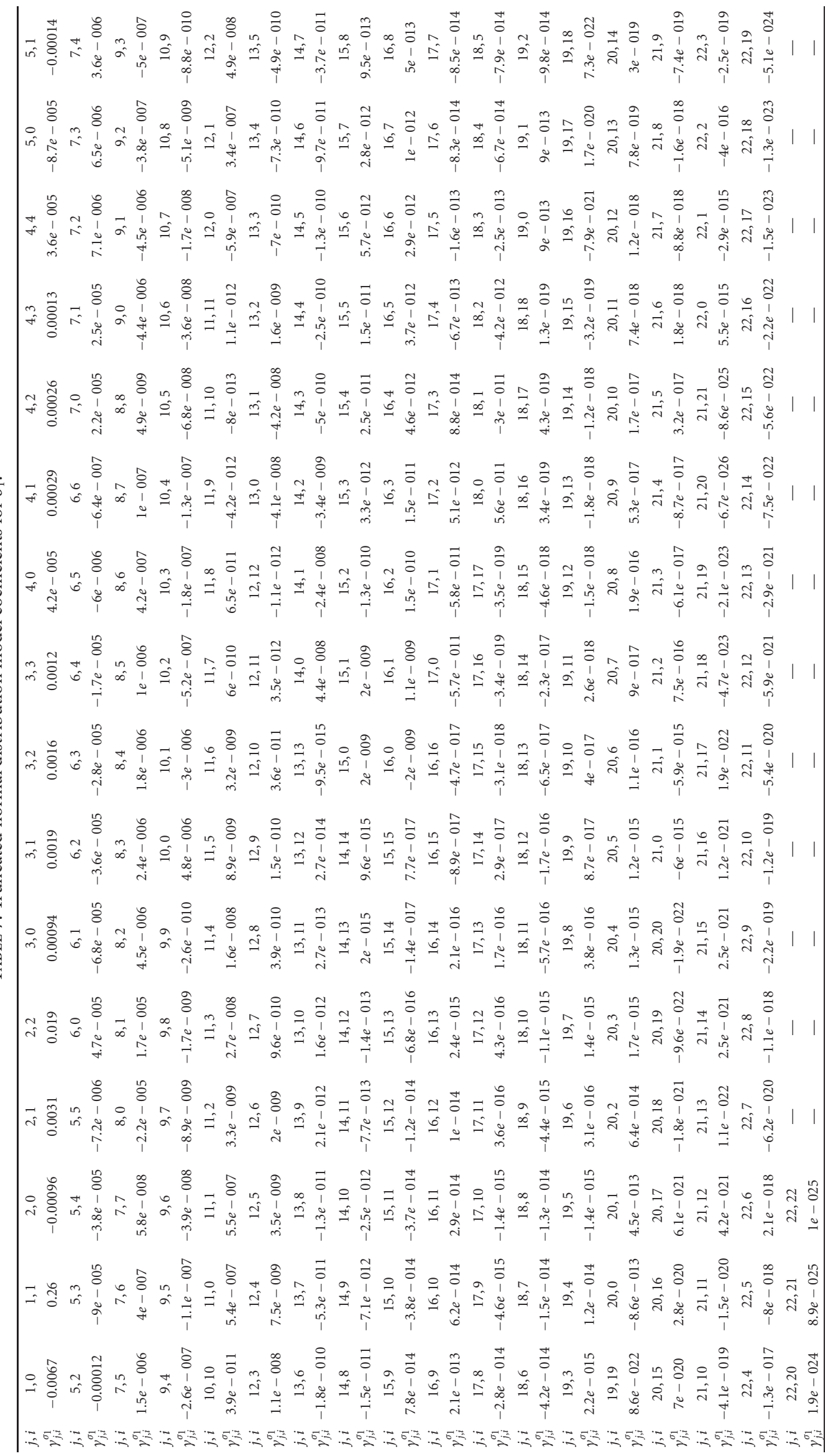


listed are the values of the correlator output, $x$, beyond which the cumulative probability density is less than $1 \times 10^{-6}$. It is clear from the table that, for signals stronger than $C / N_{0}^{D} \approx 23 \mathrm{~dB}-\mathrm{Hz}$, (the region of convergence does not contain the range of $x$-values for which the summation in (A.11) must be evaluated. Consequently, truncation of the sum without significant loss of accuracy is not possible and, hence, (A.14) is rendered useless for practical purposes. For very weak signals, $\left.C / N_{0}^{D}<23 \mathrm{~dB}-\mathrm{Hz}\right)$, numerical methods could be used in (A.14) and (A.15) to a good approximation. However, in these circumstances, Wald's approximations can be used, and these provide a much simpler approximation.

\section{B. Truncated Normal Distribution: Model Coefficients}

Tables 4, 5, 6, and 7 list the coefficients, $\gamma_{j}^{\mu_{0}}, \gamma_{j}^{\sigma_{0}}, \gamma_{j, i}^{\mu_{1}}$, and $\gamma_{j, i}^{\sigma_{1}}$, respectively, for the Taylor series models of the parameters, $\mu$ and $\sigma$, of the truncated normal approximation for the distribution of the probability ratio in the SPRT, in $H_{0}$ and $H_{1}$ cells, respectively. The $H_{0}$ coefficients are to be used in (12), whilst the $H_{1}$ coefficients refer to (9) and (11).

\section{Acknowledgements}

The Irish Research Council for Science Engineering and Technology (IRCSET) is acknowledged for funding this work, as part of the first author's doctoral studies. The authors also wish to express thanks to Dr. Michael Quinlan, of the Applied Mathematics Department. in University College Cork, for his guidance in finding functional forms for some of the expressions in this work.

\section{References}

[1] E. D. Kaplan and C. Hegarty, Understanding GPS: Principles and Applications, Artech House, 2006.

[2] A. Wald, Sequential Analysis, John Wiley \& Sons, New York, NY, USA, 2004.

[3] Y. Gong, H. Yang, W. Hu, and W. Yu, "An efficient particle filter based distributed track-before-detect algorithm for weak targets," in Proceedings of the IET International Radar Conference, pp. 1-6, April 2009.

[4] J. Guo, G. Zhong, D. Qu, and T. Jiang, "Multi-slot spectrum sensing with backward SPRT in cognitive radio networks," in Proceedings of the International Conference on Wireless Communications and Signal Processing (WCSP '09), pp. 1-5, November 2009.

[5] D. I. Kim and Y. R. Lee, "Fast cell search using I/Q multiplexed code in asynchronous DS/CDMA cellular systems," in Proceedings of the 5th IEEE International Symposium on Spread Spectrum Techniques \&amp; Applications, vol. 3, pp. 832-836, September 1998.

[6] G. S. Hosangadi and C. W. Baum, "Hybrid sequential acquisition schemes for noncoherent chip-asynchronous DS/SS systems," in Proceedings of the IEEE International Conference on Communications (ICC '98), pp. 1242-1247, Atlanta, Ga, USA, June 1998.
[7] B. Lang, T. Han, and X. Gu, "A robust non-coherent sequential code acquisition scheme for DS/SS communications," in Proceedings of the 9th International Conference on Signal Processing (ICSP '08), pp. 1939-1942, October 2008.

[8] Y. H. Lee and S. J. Kim, "Sequence acquisition of DS-CDMA systems employing gold sequences," IEEE Transactions on Vehicular Technology, vol. 49, no. 6, pp. 2397-2404, 2000.

[9] S. Tantaratana, A. W. Lam, and P. J. Vincent, "Noncoherent sequential acquisition of PN sequences for DS/SS communications with/without channel fading," IEEE Transactions on Communications, vol. 43, no. 234, pp. 1738-1745, 1995.

[10] N. O’Mahony, C. O'Driscoll, and C. C. Murphy, "Performance of sequential probability ratio test for GPS acquisition," in Proceedings of the IEEE International Conference on Communications, pp. 1-6, Dresden, Germany, June 2009.

[11] D. M. DiCarlo and C. L. Weber, "Multiple dwell serial search: performance and application to direct sequence code acquisition," IEEE Transactions on Communications, vol. 31, no. 5, pp. 650-659, 1983.

[12] P. Tong, "A suboptimum synchronization procedure for pseudo-noise communication systems," in Proceedings of the National Telecommunications Conference, pp. 26D-1-26D-5, 1973.

[13] N. O’Mahony, C. C. Murphy, and G. Lachapelle, "A dualthreshold up-down counter for GPS acquisition," Signal Processing, vol. 91, no. 5, pp. 1093-1102, 2011.

[14] K. K. Chawla and D. V. Sarwate, "Acquisition of PN sequences in chip synchronous DS/SS systems using a random sequence model and the SPRT," IEEE Transactions on Communications, vol. 42, no. 6, pp. 2325-2331, 1994.

[15] J. C. Lin, "Noncoherent sequential PN code acquisition using sliding correlation for chip-asynchronous direct-sequence spread-spectrum communications," IEEE Transactions on Communications, vol. 50, no. 4, pp. 664-676, 2002.

[16] P. Misra and P. Enge, Global Positioning Systemml: Signals, Measurements and Performance, Ganga-Jamuna Press, 2006.

[17] J. G. Proakis, "Exact distribution functions of test length for sequential processors with discrete input data," IEEE Transactions on Information Theory, vol. 9, no. 3, pp. 182-191, 1963.

[18] E. Falletti, M. Pini, L. Lo Presti, and D. Margaria, "Assessment on low complexity C/No estimators based on M-PSK signal model for GNSS receivers," in Proceedings of IEEE/ION Position Location and Navigation Symposium (PLANS '08), pp. 167-172, May 2008.

[19] M. S. Sharawi, D. M. Akos, and D. N. Aloi, "GPS C/N estimation in the presence of interference and limited quantization levels," IEEE Transactions on Aerospace and Electronic Systems, vol. 43, no. 1, pp. 227-238, 2007.

[20] C. Caini, G. E. Corazza, and A. Vanelli-Coralli, "DS-CDMA code acquisition in the presence of correlated fading-part II: application to cellular networks," IEEE Transactions on Communications, vol. 52, no. 8, pp. 1397-1407, 2004.

[21] G. E. Albert, "On the computation of the sampling characteristics of a general class of sequential decision problems," The Annals of Mathematical Statistics, vol. 25, no. 2, pp. 340-356, 1954.

[22] G. Corsini, E. Mese, G. Marchetti, and L. Verrazzani, "Comments on 'on a direct method of analysis and synthesis of the SPRT' by I. Vrana," IEEE Transactions on Information Theory, vol. 30, no. 3, pp. 573-574, 1984.

[23] W. H. Sheen and H. C. Wang, "Performance analysis of the biased square-law sequential detector with signal present," 
IEEE Transactions on Information Theory, vol. 43, no. 4, pp. 1268-1272, 1997.

[24] W. Kendall, "Performance of the biased square-law sequential detector in the absence of signal," IEEE Transactions on Information Theory, vol. 11, no. 1, pp. 83-90, 1965.

[25] M. Abramowitz and I. A. Stegun, Handbook of Mathematical Functions with Formulas Graphs and Mathematical Tables, Applied Mathematics Series, United States Department of Commerce, National Bureau of Standards, 1964.

[26] H. Akaike, "A new look at the statistical model identification," IEEE Transactions on Automatic Control, vol. 19, no. 6, pp. 716-723, 1974.

[27] A. K. Seghouane, "The akaike information criterion with parameter uncertainty," in Proceedings of the 4th IEEE Sensor Array and Multichannel Signal Processing Workshop (SAM '06), pp. 430-434, Waltham, Mass, USA, July 2006.

[28] PLAN Group, “GSNRx ${ }^{\mathrm{TM}}$ receiver," 2010, http://plan.geomatics.ucalgary.ca/software_gsnrx.php.

[29] J. G. Proakis and M. Salehi, Digital Communications, McGrawHill, 5th edition, 2008. 

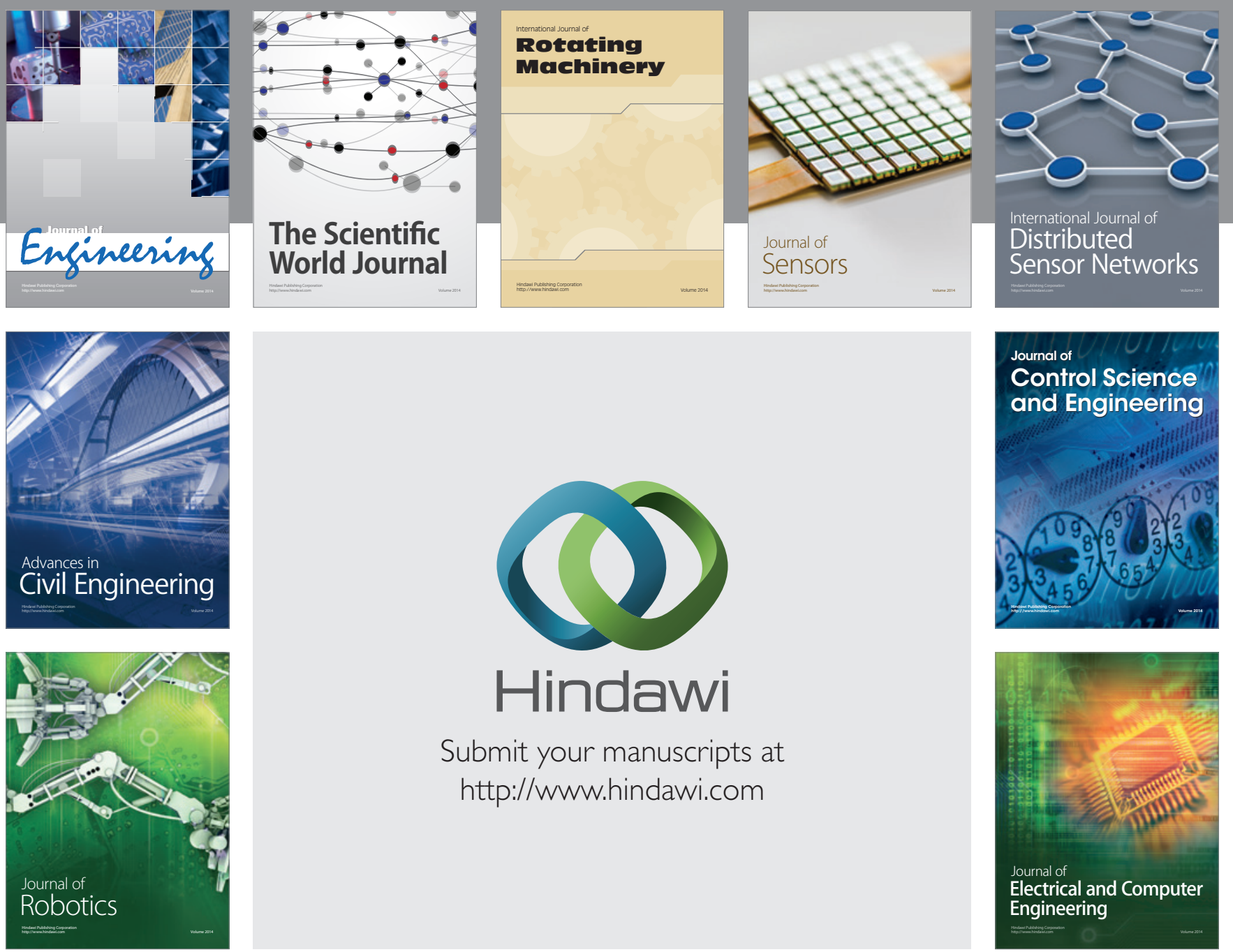

Submit your manuscripts at

http://www.hindawi.com
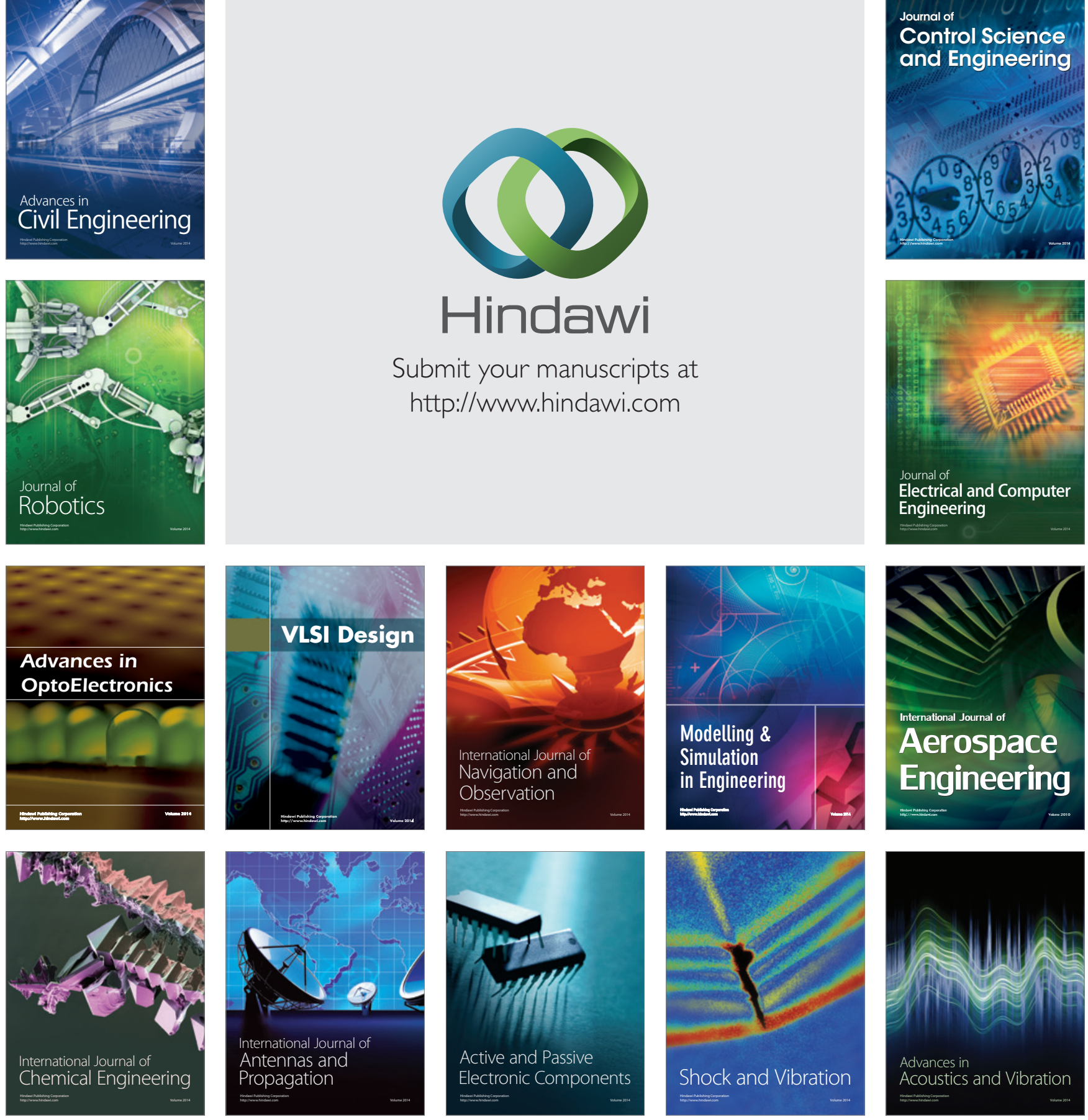